\title{
Design and testing of a four rod crab cavity for High Luminosity LHC
}

\author{
B. Hall, ${ }^{\dagger}$ G. Burt, R. Apsimon, ${ }^{*}$ C. J. Lingwood, and A. Tutte \\ Engineering Department, Lancaster University, Lancaster LA1 4YW, United Kingdom \\ and Cockcroft Institute, Daresbury Laboratory, Warrington WA4 4AD, United Kingdom
}

A. Grudiev, A. Macpherson, M. Navarro-Tapia, and R. Calaga

CERN, Geneva CH-1211, Switzerland

K. G. Hernández-Chahín

DCI-UG, Guanajuato 37150, Mexico

R. B. Appleby

School of Physics and Astronomy, The University of Manchester, Manchester M13 9PL, United Kingdom and Cockcroft Institute, Daresbury Laboratory, Warrington WA4 4AD, United Kingdom

P. Goudket

STFC Daresbury Laboratory, Warrington WA4 4AD, United Kingdom

(Received 22 June 2016; published 3 January 2017)

\begin{abstract}
A 4-rod deflecting structure is proposed as a possible crab cavity design for the LHC high luminosity upgrade. Crab cavities are required for the LHC luminosity upgrade to provide a greater bunch overlap in the presence of a crossing angle, but must fit in the existing limited space. The structure has two parallel sections consisting of two longitudinally opposing quarter-wave rods, where each rod has the opposite charge from each of its nearest neighbors. The structure is transversely compact because the frequency is dependent on the rod lengths rather than the cavity radius. Simulations were undertaken to investigate the effect of rod shape on surface fields, higher order multipole terms and induced wakefields in order to obtain the optimal rod shape. The simulation results presented show that the addition of focus electrodes or by shaping the rods the sextupole contribution of the cavity voltage can be negated; the sextupole contribution is $321.57 \mathrm{mTm} / \mathrm{m}^{2}, \mathrm{E}_{\text {peak }}=27.7 \mathrm{MV} / \mathrm{m}$, and $\mathrm{B}_{\text {peak }}=63.9 \mathrm{mT}$ at the design voltage of $3 \mathrm{MV}$. The damping requirements for the LHC are critical and suitable couplers to damp all modes but the operating mode are presented. The results of various testing cycles of the first SRF 4 rod prototype cavity are presented and show that the cavity has reached the required transverse voltage of $3 \mathrm{MV}$.
\end{abstract}

DOI: 10.1103/PhysRevAccelBeams.20.012001

\section{INTRODUCTION}

The LHC will be upgraded to increase the luminosity by a factor of 10 during the third long shutdown (LS3); which is planned for 2023-2025. The LHC high luminosity upgrade (HL-LHC) [1] project will increase luminosity by reducing the beam sizes in the interaction regions. However this requires a larger crossing angle to reduce long-range beambeam effects and an increase in crossing angle coupled with the smaller beam width results in a decrease in luminosity because the beams no longer fully overlap. It should also be noted that the crossing angle is the angle of intersection between the counterrotating beams at the interaction point

\footnotetext{
"r.apsimon@lancaster.ac.uk

Present address: HZB, Berlin, Germany.
}

Published by the American Physical Society under the terms of the Creative Commons Attribution 3.0 License. Further distribution of this work must maintain attribution to the author $(s)$ and the published article's title, journal citation, and DOI.
(IP) and not the bunch rotation angle. To mitigate against this luminosity loss, the bunches transverse momentum is chirped longitudinally in the horizontal or vertical plane by applying a time-varying transverse kick to the bunches such that the bunches completely overlap at the IP.

The time-varying transverse kick required to apply a longitudinal chirp to the bunch is achieved with transverse deflecting cavities, known as crab cavities [2]. Crab cavities operate in a dipole mode and produce the required transverse deflection with transverse electric and magnetic fields. The electric and magnetic fields for a four rod crab cavity (4RCC) are shown in Fig. 1. Crab cavities do not differ from conventional transverse deflecting cavities except that they operate with a 90 degree phase shift such that the centroid of the bunch experiences no net deflection through a crab cavity; while the head and tail experience equal and opposite kicks in order to crab the bunch.

To minimize the required transverse voltage the cavities are installed in a region with a large $\beta$ function and at a 


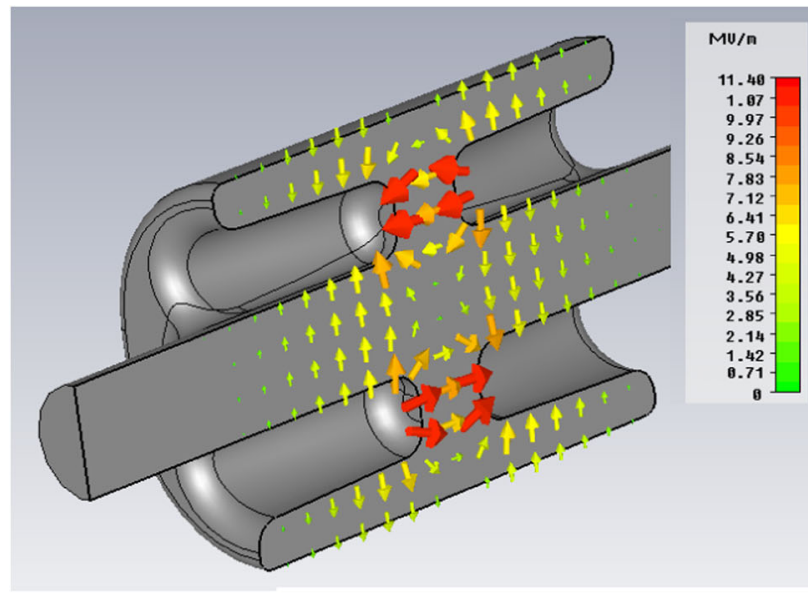

(a)

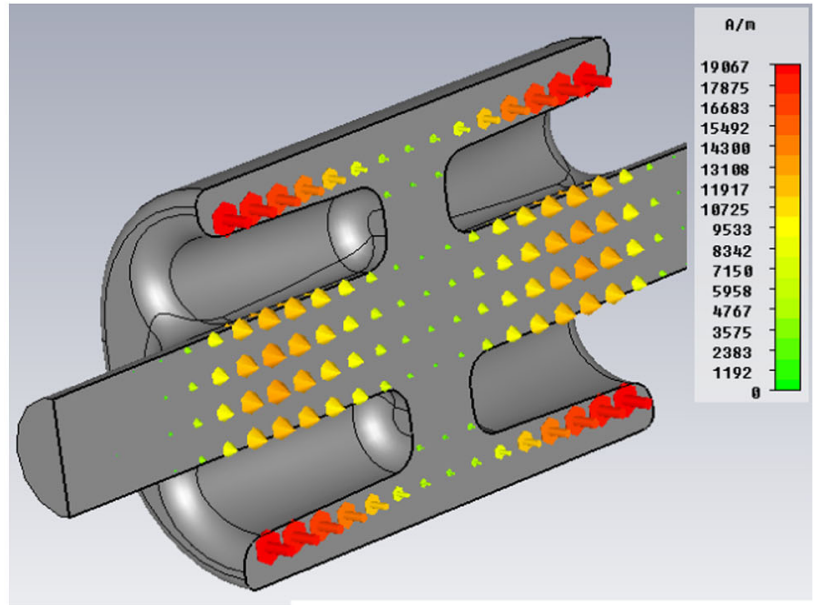

(b)

FIG. 1. Electric (a) and magnetic (b) fields (arbitrary units) in a 4RCC.

phase advance of $\pi / 2$ upstream of the IP. In order to close the crab bump a second set of cavities are installed at a phase advance of $\pi / 2$ downstream of the IP. At the time of the design study described in this paper, the required transverse voltage to fully crab the beam was $10-12 \mathrm{MV}$, however this value is still changing, but will be considered as $12 \mathrm{MV}$ for consistency in this paper. To achieve $12 \mathrm{MV}$, 4 cavities are required either side of the interaction point (IP) for each beam at each IP. Such a high voltage could not be sustained in continuous wave without the use of a superconducting rf (SRF) deflecting cavity.

The first SRF deflecting cavity was developed at Karlsruhe, in collaboration with CERN, and was installed in 1977 [3]. Crab cavities have also been demonstrated at the KEKB electron-positron collider [4]. The KEKB crab cavities operate in a $\mathrm{TM}_{110}$-like mode of an elliptical cavity and use a racetrack cross section to separate the mode polarizations. This system showed the expected increase in luminosity at low beam currents; however such a concept has never been demonstrated on hadron beams.
The RMS bunch length of the LHC bunches is $7.55 \mathrm{~cm}$ and dictates the frequency range for the crabbing system. The frequency of the crab cavity must be a harmonic of the $40 \mathrm{MHz}$ bunch repetition frequency. If the chosen harmonic is too high then the transverse kick to the bunch will not vary linearly with longitudinal position across the bunch length. However if the frequency is too low then an excessively high rf voltage is required to achieve the desired deflection along the bunch length; $400 \mathrm{MHz}$ has been selected as a suitable compromise. The transverse voltage of a crab cavity, $V_{T}$ can be expressed as

$$
V_{T}=\frac{c^{2} p_{s} \tan \left(\frac{\theta}{2}\right)}{q \omega \sqrt{\beta^{*} \beta_{\text {crab }}} \sin \left(\Delta \phi_{0}\right)}
$$

where $c$ is the speed of light, $p_{s}$ is the particle momentum, $\theta$ is the crossing angle, $q$ is the charge of the particle, $\omega$ is the angular frequency of the cavity, $\Delta \phi_{0}$ is the betatron phase advance between the crab cavity and the IP, and $\beta^{*}$ and $\beta_{\text {crab }}$ are the values of the $\beta$ function at the IP and crab cavity location, respectively.

The transverse gap between the counterrotating beams in the LHC tunnel puts stringent constraints on the dimensions of the crab cavity. In the proposed location for the crab cavity, the beams are separated by $194 \mathrm{~mm}$ and the diameter of the opposing beam pipe is $84 \mathrm{~mm}$; this provides $152 \mathrm{~mm}$ [5] for the crab cavity including the space for cryogenic shielding, thermal contraction and the cavity wall thickness. Thus the inner radius of the outer can of the crab cavity will be limited to $145 \mathrm{~mm}$ for all designs outlined in this paper to meet these spatial requirements. At $400 \mathrm{MHz}$ an elliptical cavity similar to the KEKB cavity would have a radius more than double this and hence a novel compact design is required to meet the stringent LHC space requirements. The cavity design should preferably be compact both horizontally and vertically to allow the same cavity design to be used to crab the beam horizontally or vertically to recover the luminosity loss due to the beam crossing angle, although this is not a requirement as a different cavity could be used for each crossing plane. The need for the two colliding beams to share a common beampipe around the interaction region results in additional bunch-bunch interactions due to long-range Coulomb interactions; this is referred to as long-range beam-beam interaction. This breaks the symmetry between the two transverse planes, couples horizontal and vertical motion and generates tune shift, of opposite sign, to the head-on collision. This additional tune-shift depends on the crossing plane, so the overall tune-shift can be minimized by alternating the crossing planes, so the head-on tune shift is partially compensated in both planes. Hence the need for both horizontal and vertical crab cavity deflection. The cavity should have an aperture of $84 \mathrm{~mm}$ and should be capable of providing a transverse voltage of $3 \mathrm{MV}$ [Eq. (1)]. 
The LHC 4RCC, designed at the Cockcroft Institute, is proposed here as a possible solution for a compact crab cavity design. The 4RCC is based on a concept similar to the normal conducting Continuous Electron Beam Accelerator Facility (CEBAF) deflector [6,7], where the cavity is operated using the TEM dipole mode of four quarter wave rods inside an outer conductor. The structure has four quarter wavelength rods, consisting of two parallel rods being mirrored in the longitudinal plane as shown in Fig. 2. Each rod has the opposite potential from its transverse and longitudinal neighbor for the crabbing mode, but the structure has four modes closely spaced in frequency for the four different combinations of rod polarity. Such a cavity has been designed to meet the stringent HL-LHC spatial requirements initially modify the CEBAF design by increasing the beam pipe radius and rod size SRF operation in the LHC. The CEBAF cavity has an aperture of $15 \mathrm{~mm}$ which is too small for the LHC. Collimation studies have suggested a minimum aperture size is $84 \mathrm{~mm}$ hence the aperture is required to be opened up.

Microphonics is the term used to describe the excitation of a structures mechanical resonances by external vibrations. This can lead to small vibrational forces leading to relatively significant displacements, which shifts the cavity frequency by an appreciable fraction of its bandwidth. In superconducting radio-frequency (SRF) cavities, due to the narrow bandwidth any small vibrations that cause frequency shifts larger than this bandwidth can cause problems with field stability. The normal conducting CEBAF cavity has a rod radius of only $10 \mathrm{~mm}$, the flexibility of the rods would cause significant vibrations, leading to microphonics, in a SRF variant of this cavity; thus a larger rod radius is required. There are also additional constraints on the maximum cavity size, as detailed above and more stringent performance requirements in terms of the peak surface electric and magnetic fields.

Due to the lack of azimuthal symmetry the deflecting field is not a pure dipole and contains many higher order multipolar components. This results in a transverse deflecting voltage that varies with particle transverse position which reduces the dynamic aperture of the LHC.

Two other designs of compact crab cavities are also proposed for LHC, the double-quarter wave [8] and rf dipole $[9,10]$. This paper will focus only on the $4 \mathrm{RCC}$ design.

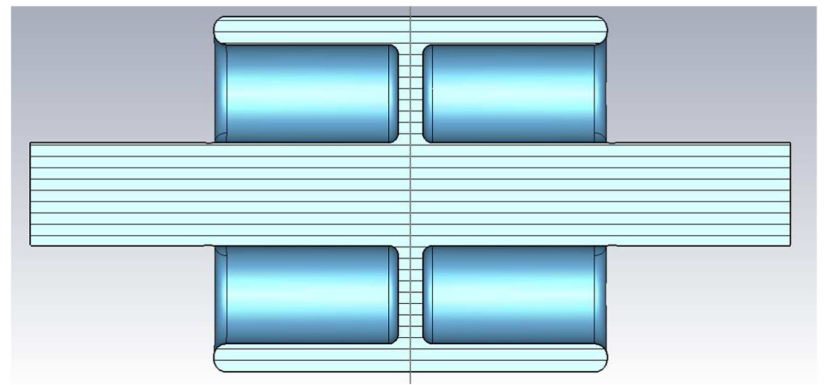

FIG. 2. A cross sectional diagram of a 4 RCC.
In Sec. II of this paper we will present the design of a cylindrical 4RCC. By considering conical elliptical rods, we investigate the dependence of the base and tip ellipse dimensions on peak surface fields and transverse shunt impedance in the cavity. In Sec. III, we consider the higher order multipole field components of the cavity and their impact on the beam dynamics in the LHC, showing the sextupole component is the largest concern. In Secs. III A and III B we show how focus electrodes (wings) and kidney-shaped rods can be designed to negate these sextupole fields in the cavity; kidney-shaped rods are selected for the final design as they allow for lower peak surface fields than wing-shaped rods and they are easier to machine. We focus on correcting the sextupole term because there is no quadrupole or octupole term due to the symmetry of the cavity and the decapole term is found to be negligible for expected transverse offsets in the 4RCC. In Sec. III C we summarize the design parameters for different prototype designs. Bead pull measurements were taken of a prototype crab cavity in order to study if the sextupole component can be measured and the results are shown in Sec. IV. Section V shows couplers that would be suitable for use in the LHC to damp all the modes that are not the operating mode and the cavity impedance is presented. In Sec. VI the results of the first high gradient cold tests of a SRF 4 rod deflecting structure are presented. The conclusions are presented in Sec. VII.

\section{OPTIMIZATION OF A 4 ROD CAVITY}

In order to achieve the required transverse voltage of $3 \mathrm{MV}$, a transverse gradient of $9 \mathrm{MV} / \mathrm{m}$ is required for a crab cavity which is $\lambda / 2$ in length at $400 \mathrm{MHz}$. To obtain this gradient in stable operation the peak surface fields must be limited. While no fundamental limits exist we choose in this study to limit the fields to $E_{\text {peak }}<50 \mathrm{MV} / \mathrm{m}$ and $B_{\text {peak }}<100 \mathrm{mT}$, based on cold tests of other complex SRF structures. The magnetic field is a conservative limit based on a review of cold test results from cavities with complex geometries. The ratio of $B_{\text {peak }} / E_{\text {peak }}$ was chosen to be 2 , while other designers tend to opt for ratios of 1.5-1.8 [9], it was decided that $E_{\text {peak }}$ should be lower due to the difficultly of the high pressure rinse on the complex rod geometry.

For the optimization of the cavity design, the peak fields will be divided by the transverse voltage, $V_{T}$, to provide quantities which are independent of the energy stored in the cavity. The transverse voltage can be calculated from the longitudinal voltage using the Panofsky-Wenzel theorem [11]

$$
V_{T}=-\frac{i c}{\omega} \nabla_{T} V_{z}
$$

where $\omega$ is the angular frequency of the cavity, $c$ is the speed of light, and $\nabla_{T} V_{z}$ is the transverse gradient of the longitudinal voltage. In addition to minimization of the peak surface fields, it is also important to minimize the dynamic 
cryogenic load of the cavity. This is characterized by the cavity transverse shunt impedance,

$$
R_{T}=\frac{V_{T}^{2}}{2 P}=\frac{G}{2 R_{S}} \frac{R}{Q}
$$

where $G=Q_{0} R_{S}$ is the geometric factor, $P$ is the power dissipated as heat in the cavity walls due to ohmic heating, and $R_{S}$ is the surface resistance of the cavity walls. However as $R_{T}$ is inversely proportional to the cavity surface resistance, it is conventional to optimize the cavity geometry to minimize the product $\frac{G}{2} \frac{R}{Q}$, which is independent of the surface resistance.

\section{A. Conical elliptical rods}

The design of the 4RCC for the LHC is loosely based on the CEBAF deflecting cavity [7]; however several design changes are required for LHC. First the rods must be separated significantly to allow for the required aperture in LHC and the rods must be made wider to stiffen them against microphonics. There are additional constraints on the maximum cavity size and more stringent performance requirements in terms of the peak surface electric and magnetic fields.

The 4RCC geometry can be characterized by a number of parameters, depending on the cavity design. For a conical elliptical rod shape, the tip and base of the rods are parametrized by the width and breadth of each ellipse as well as the gap between rods and the length of the rods (Fig. 3). The length of the rods has the strongest effect on the resonant frequency and is varied to maintain a cavity frequency of $400 \mathrm{MHz}$ as the other parameters are varied [12]. The cavity was optimized in CST Microwave Studio [13] with 200000-400000 tetrahedral mesh cells per simulation.

As the gap decreases the transverse voltage also decreases for fixed values of peak surface fields because the electric fields around the rods also decreases (Fig. 4); hence so does the electric field along the beam axis. From the Panofsky-Wenzel theorem the transverse voltage is seen to be directly proportional to the change in longitudinal voltage with transverse position. Since $V_{z}(r)$ depends on the size of the longitudinal gap, hence so does $V_{T}$. As we are focused on small gaps compared to the wavelength, the transit time factor is close to unity and can therefore be neglected. Hence reducing the fringe fields near the beam will also reduce the transverse voltage; hence the gap should be large enough to provide sufficient transverse voltage. However an excessive gap length reduces the transverse shunt impedance in the cavity [12], thus the gap will be fixed at $60 \mathrm{~mm}$ for all further simulations. $B_{\text {peak }} / V_{T}$ is not affected by the longitudinal rod gap because there is no magnetic field in this region; this has also been confirmed with simulations. The longitudinal rod gap affects the relative phase between the E- and B-fields, which in turn affects $V_{T}$ and also affects $E_{\text {peak }} / V_{T}$.

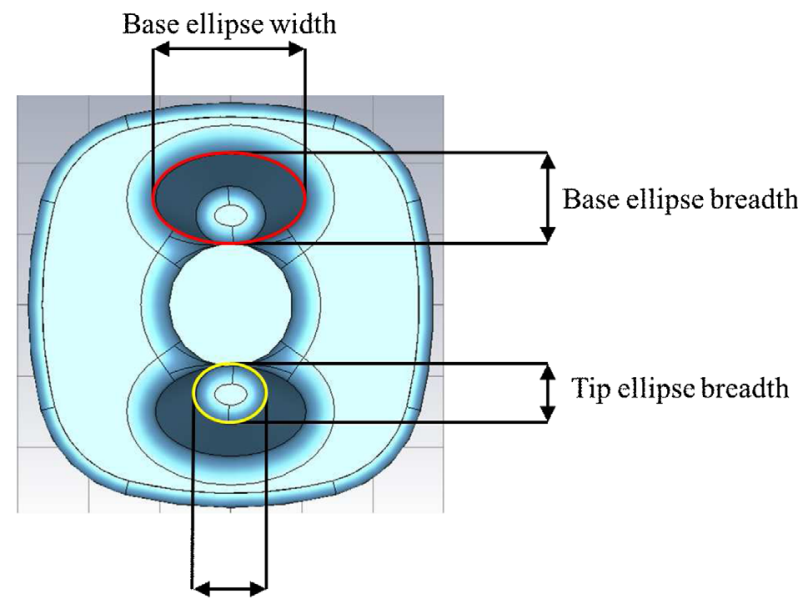

Tip ellipse width

(a)

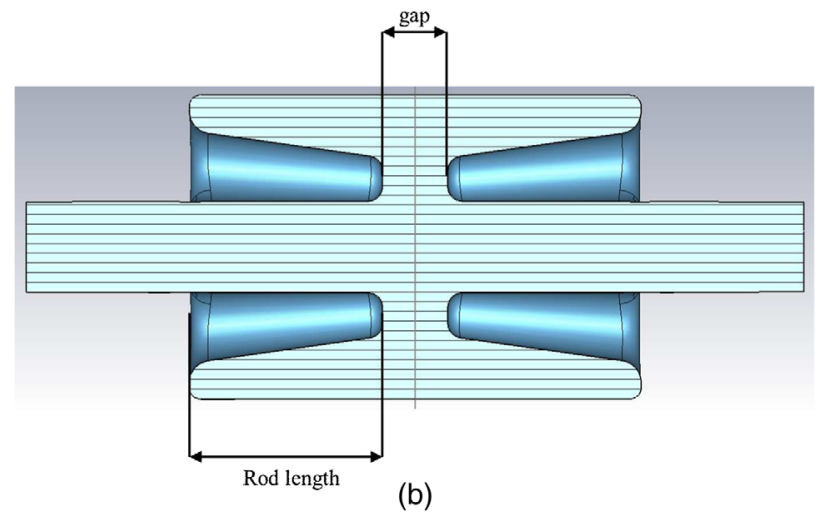

FIG. 3. Diagrams of the conical elliptical 4RCC, viewed along the beam axis (a) and a transverse cross-section (b) to show the parameterization of the rod design parameters.

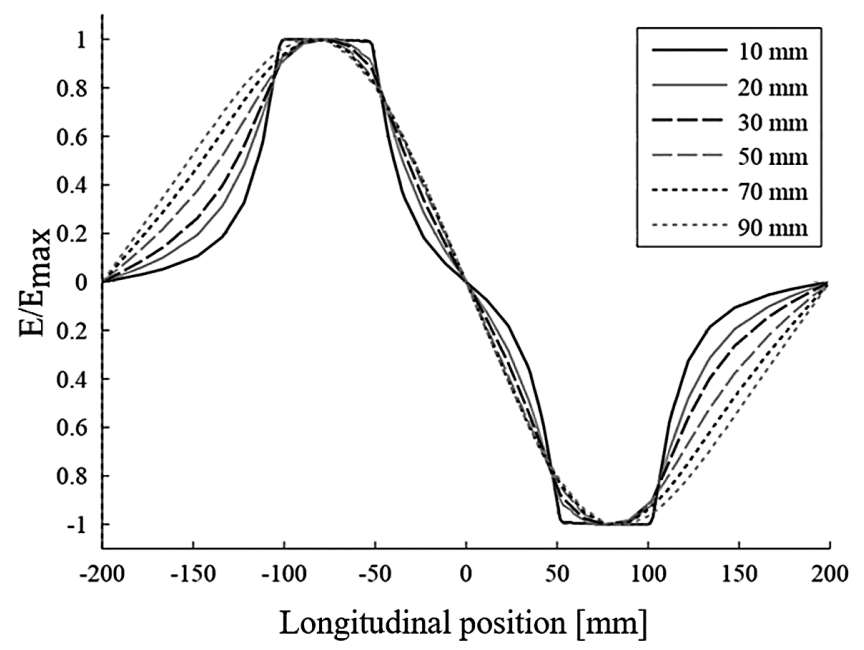

FIG. 4. Fringe transverse electric fields vs longitudinal position for different rod gaps. 
From Fig. 1, it can be seen that the electric field is concentrated around the tip and the magnetic field is concentrated around the base; thus the peak electric and magnetic fields can be minimized independently by varying the tip and base ellipse parameters, respectively. The base ellipse rounding was set to $20 \mathrm{~mm}$ for these simulations as it is sufficient prevent large peak magnetic fields near the rod base.

We begin by optimizing the base ellipse. Figure 5 shows $B_{\text {peak }} / V_{T}$ vs base width for different values of base breadth. As the base width is increased, the peak magnetic field decreases and reaches a plateau for widths larger than $110 \mathrm{~mm}$; this is because the surface current has to flow around the beam pipe which is $84 \mathrm{~mm}$ in diameter. Similarly as the base breadth is increased, the peak magnetic field decreases and reaches a plateau for breadths larger than $65 \mathrm{~mm}$.

Figure 6 shows $\frac{G}{2} \frac{R}{Q}$ vs base width for different values of base breadth. As can be seen from the plot, the transverse shunt impedance is maximized for a base breadth of $110 \mathrm{~mm}$ and decreases with base width. The base ellipse dimensions do not significantly affect the transverse voltage, but it does affect the peak surface magnetic field; which in turn affects the rf losses in the cavity. Thus an increase in $B_{\text {peak }}$ will result in a reduction in transverse shunt impedance. A base width of $110 \mathrm{~mm}$ and breadth of $65 \mathrm{~mm}$ has been selected as a compromise between minimizing the peak surface magnetic field and maximizing the transverse shunt impedance.

$\frac{G}{2} \frac{R}{Q}$ vs tip width for different values of tip breadth was also simulated. This showed that the transverse shunt impedance is maximized when the tip ellipse width and breadth are both minimized; however this will lead to a large value of $E_{\text {peak }}$. A tip width and breadth of $45 \mathrm{~mm}$ and $40 \mathrm{~mm}$, respectively, have been chosen as the optimal working point.

A similar optimization was undertaken for the tip ellipse. The tip rounding was set to the minor axis of the tip to

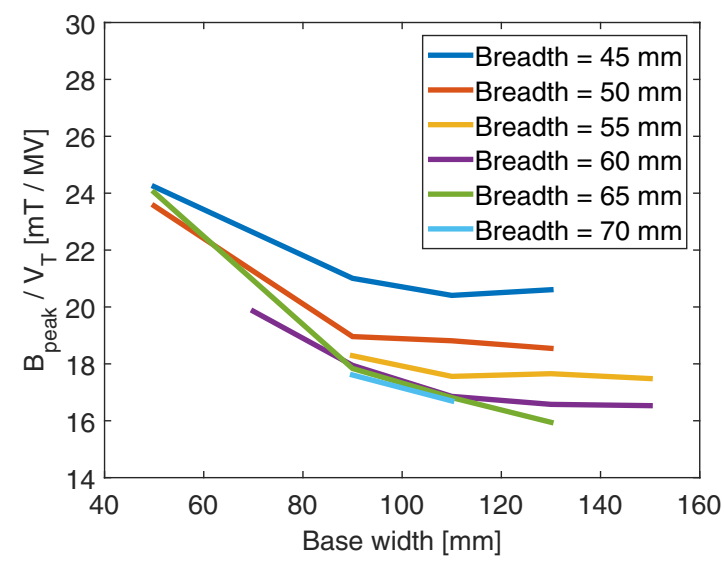

FIG. 5. $B_{\text {peak }} / V_{T}$ vs base width for different values of base breadth. The tip width and breadth were fixed at $45 \mathrm{~mm}$ and $40 \mathrm{~mm}$, respectively.

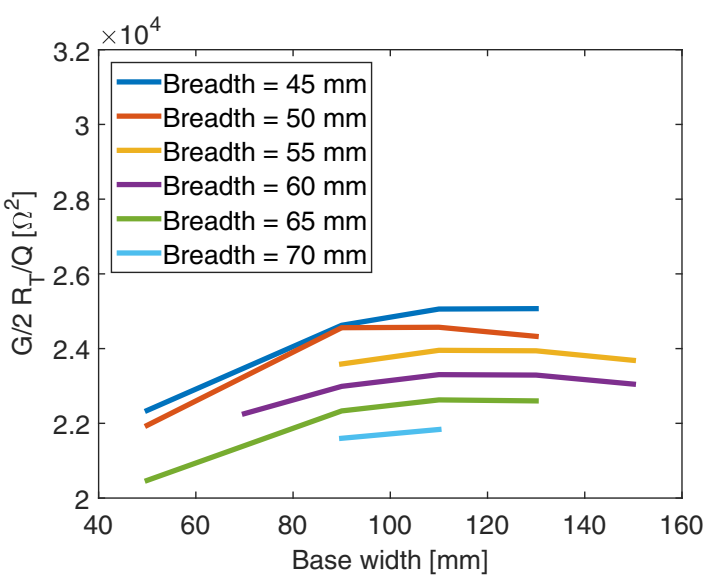

FIG. 6. $\frac{G}{2} \frac{R}{O}$ vs base width for different values of base breadth. The tip width and breadth were fixed at $45 \mathrm{~mm}$ and $40 \mathrm{~mm}$, respectively.

minimize the curvature. Varying the tip geometry does not affect the peak surface magnetic field but as it affects $V_{T}$, it is important to monitor $B_{\text {peak }} / V_{T}$ (Fig. 7). As can be seen, $B_{\text {peak }} / V_{T}$ has little dependence on the tip breadth $<50 \mathrm{~mm}$ and increases slightly with tip width; this is because as the tip dimensions increase, $V_{T}$ decreases.

The tip geometry directly affects the transverse electric field, thus $V_{T}$. The transit time between the base of the rod, where the magnetic field is situated, and the tip of the rod, where the electric field is situated, applies a small phase shift between the magnetic and electric fields; which results in a change in $V_{T}$.

Figure 8 shows $E_{\text {peak }} / V_{T}$ vs tip width for different values of tip breadth. If the tip breadth is too small the surface electric field concentrates at the tip and $E_{\text {peak }} / V_{T}$ reaches a plateau for tip breadths $\geq 40 \mathrm{~mm}$ due to localized field enhancement. Similarly, as the width increases $E_{\text {peak }} / V_{T}$ decreases and reaches a plateau for tip widths $\geq 45 \mathrm{~mm}$.

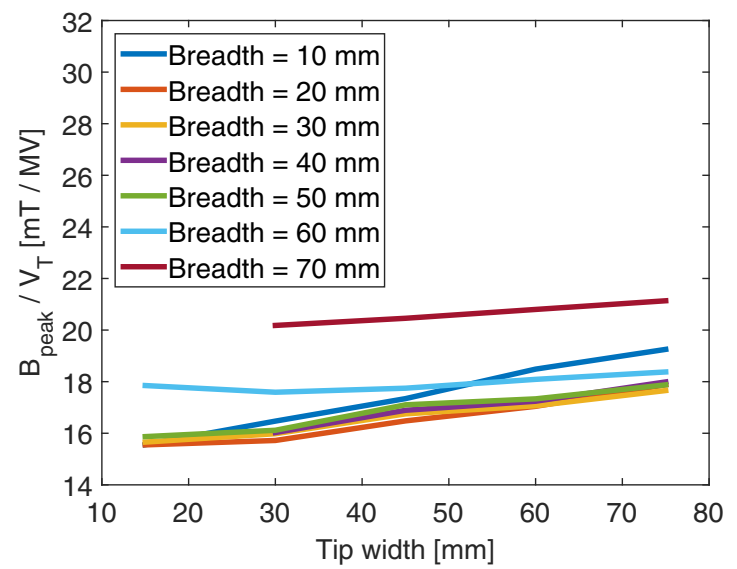

FIG. 7. $B_{\text {peak }} / V_{T}$ vs tip width for different values of tip breadth. The base width and breadth were fixed at $110 \mathrm{~mm}$ and $65 \mathrm{~mm}$, respectively. 


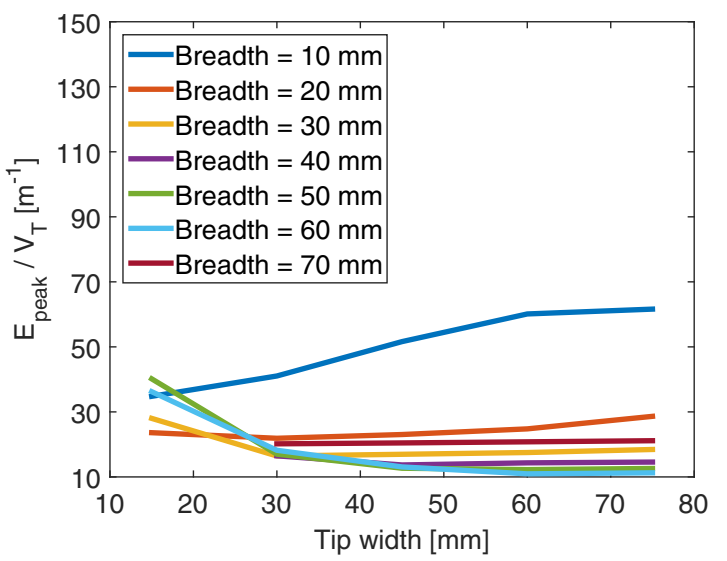

FIG. 8. $E_{\text {peak }} / V_{T}$ vs tip width for different values of tip breadth. The base width and breadth were fixed at $110 \mathrm{~mm}$ and $65 \mathrm{~mm}$, respectively.

In addition to the main crabbing mode, it is also important to consider other modes with frequencies near $400 \mathrm{MHz}$. Indeed due to the symmetry of the crab cavity, there are two dipole modes and two monopole modes with frequencies near $400 \mathrm{MHz}$; excitation of these other modes by passing proton bunches will lead to wakefields in the cavity which will affect later bunches. The second dipole mode will lead to a transverse kick to the centroid of the beam; however this mode has equal charges on each longitudinally opposing rod hence has low longitudinal electric field and is not excited by the beam. For the monopole modes, one of the modes will have the same charge on all four rods and is not excited by the beam, the other monopole mode has a net longitudinal voltage which will introduce energy deviations to the bunches. Sketches of the rod polarity configurations for the monopole and dipole modes are shown in Figure 9.

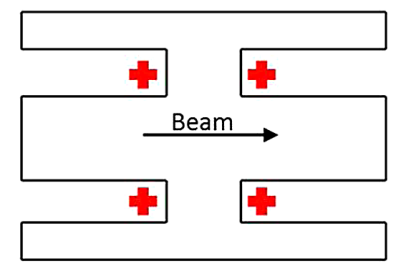

(a)

Monopole

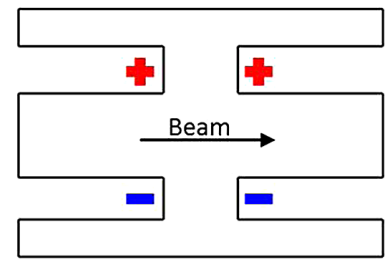

(c)

Dipole

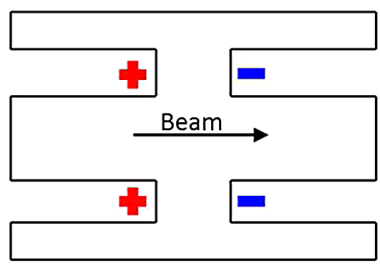

(b)

Monopole (LOM)

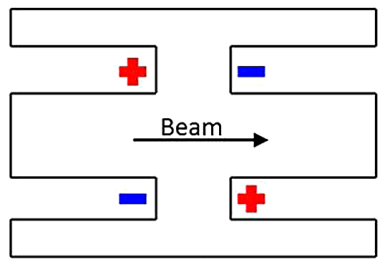

(d)

Dipole (crab)
FIG. 9. Sketches of the rod polarity configurations for the monopole and dipole modes in a 4RCC.
By considering the monopole mode which is excited by the beam, we can estimate the effect of wakefields in the cavity. Due to the capacitance between the rods, this mode is always at a lower frequency than the dipole mode and is known as the lower order mode (LOM) [12]. The LOM $\mathrm{R} / \mathrm{Q}$ vs base width and breadth is shown in Fig. 10. As the base breadth increases the LOM R/Q decreases; thus a larger rod breadth is beneficial. This is because as the fields are concentrated around the rods, an increase in the surface area increases the stored energy and hence decreases the $\mathrm{R} / \mathrm{Q}$. However the LOM R/Q is maximized for a base width of $110 \mathrm{~mm}$, which is the design value; but the increase in $\mathrm{R} / \mathrm{Q}$ is considered acceptable in comparison to the increase in $B_{\text {peak }} / V_{T}$ resulting from a decrease in base width. The same simulation was run while varying the tip ellipse dimensions which shows that the LOM R/Q is very weakly dependent on the tip width and is minimized when the tip breadth is as large as possible.

The dimensions of the outer can affect the LOM R/Q as well as $\mathrm{R}_{T} / \mathrm{Q}$ for the crabbing mode; as shown in Fig. 11. A cylindrical $4 \mathrm{RCC}$ design was used for this parameter scan in order to illustrate the dependence of R/Q on outer can radius. The range of values for the outer can radius exceeds the spatial constraint for the crab cavity stated in the introduction; this has been done in order to look at the $\mathrm{R} / \mathrm{Q}$ dependence over a larger range in order to be applicable for more generic $4 \mathrm{RCC}$ designs. The can radius for the final 4RCC design are of course within the specified limits.

Figure 12 shows the LOM frequency vs width for different base and tip breadths. For the base ellipse, the LOM frequency is minimized when the width is $110 \mathrm{~mm}$ and increases as the breadth increases. For the tip ellipse, the LOM frequency decreases as the tip width increases and the frequency is minimized when the tip breadth is $\sim 40 \mathrm{~mm}$ as the capacitance at the end of the rod is increased. The length of the rods is strongly coupled to

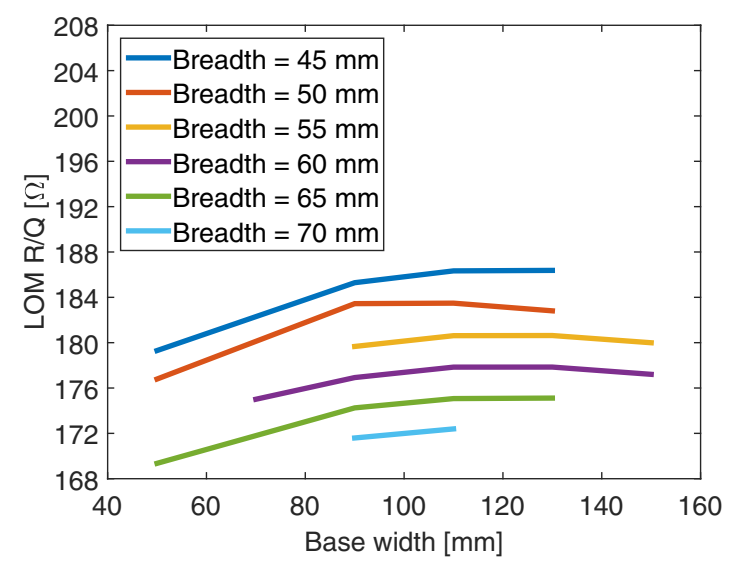

FIG. 10. R/Q of the LOM vs width for different values of breadth for the base ellipse with the tip width and breadth fixed at $45 \mathrm{~mm}$ and $40 \mathrm{~mm}$, respectively. 


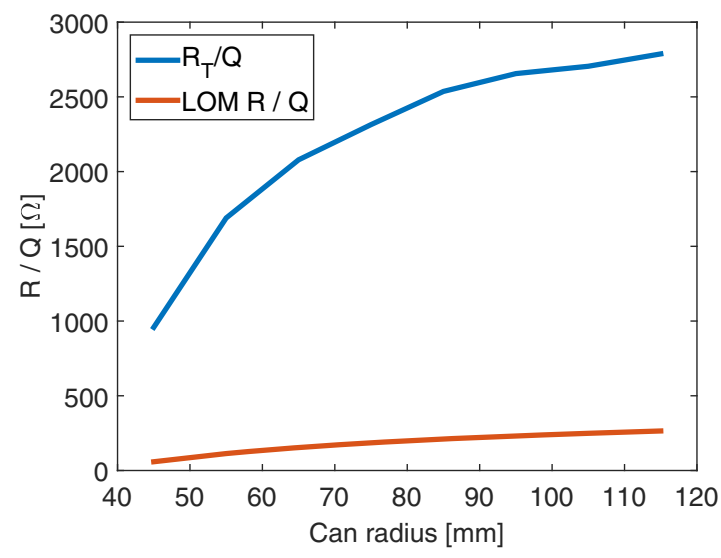

FIG. 11. Plot of the $\mathrm{R}_{T} / \mathrm{Q}$ (blue) and LOM R/Q (red) vs. radius for the outer can.

the frequency of the crab mode, whereas the dimensions of the outer can of the cavity is strongly coupled to the frequency of the LOM. Therefore we can tune the LOM frequency such that it is sufficiently far from the crabbing mode to avoid induced wakefields, whilst also sufficiently far from a harmonic of the bunch repetition frequency to avoid resonant effects.

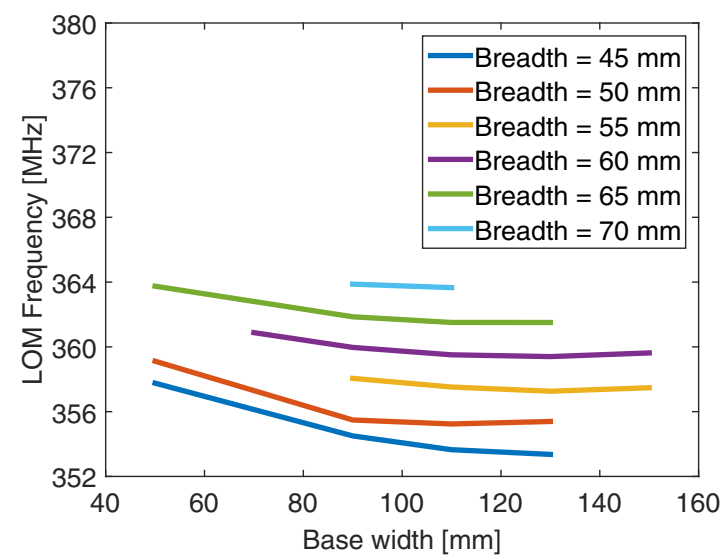

(a)

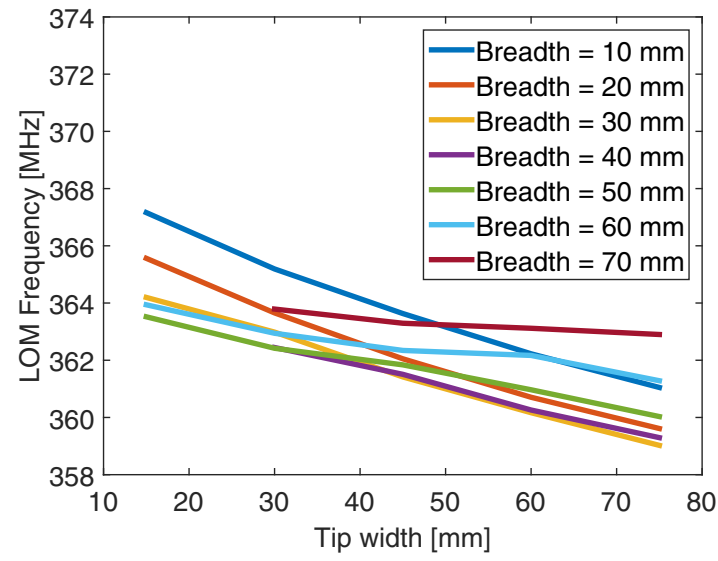

(b)

FIG. 12. Frequency of the LOM vs width for different values of breadth for the base (a) and tip (b) ellipses.

\section{MULTIPOLE EXPANSION AND BEAM DYNAMICS}

The dipole mode of complex cavity geometries is often not a pure dipole mode and the azimuthal variation in the longitudinal electric field will often have monopole, dipole, quadrupole, and sextupole components. The multipole expansion of a rf field can be written as

$$
V_{z}(\theta, r)=\sum_{n} a_{n} r^{n} \sin (n \theta)+b_{n} r^{n} \cos (n \theta),
$$

where $b_{n}$ describes the normal multipole components. In the 4RCC, due to symmetry, only odd multipole components are possible and hence we are only concerned with dipole, sextupole, and decapole terms. The addition of couplers and manufacturing errors may also give rise to even multipolar terms but these are neglected in this paper.

We can describe the impact of the crab cavity on the beam dynamics of the machine in a variety of approximations. The simplest method is inspired from the multipole description of magneto-static devices and models the effect of the cavity using a series of normal and skew integrated multipoles in the ultrarelativistic and kick approximations. The multipoles for the LHC crab cavities are under study in $[14,15]$ in the context of the machine dynamic aperture. An alternative approach using Taylor maps was successfully used in this context for the LHC in $[14,16]$ and for accelerating rf cavities in [17]. The transverse symmetries of the cavities restrict the allowed multipoles, with the behavior of the multipoles following the corresponding magnet behavior. Hence the dipolar cavities will only exhibit odd normal multipoles, with even multiples and skew multipoles being only nonzero in the presence of numerical noise. Therefore the first allowed multipole is a sextupole for a perfect cavity. The limit on the allowed sextupole is complex and is a function of the machine optics and dynamical aperture considerations. Stability studies have been undertaken and suggest that the sextupole term should not exceed $\sim 1500 \mathrm{mTm} / \mathrm{m}^{2}$ in order to allow an orbit tolerance of $>1 \mathrm{~mm}$ and negligible impact on the dynamic aperture [18]. Furthermore the dynamic aperture of HL-LHC has been studied in [14,15] assuming a $b_{3}$ of $1159 \mathrm{mTm} / \mathrm{m}^{2}$ and found no significant impact on the LHC dynamic aperture.

In a $4 \mathrm{RCC}$, if the rods were infinitely wide, the rods would become infinite parallel plates; thus producing a pure dipole field. However as the rod width decreases, the magnitude of the sextupole field increases for a fixed dipole field of $33.3 \mathrm{mT}$ (Fig. 13); the decapole components of the cavities are of the order of $0.1-5 \times 10^{6} \mathrm{mTm} / \mathrm{m}^{4}$.

The impact of the crab cavities on the beam up to octupole ( $b_{4}$ in multipole notation) has been carefully studied in [15], showing a large tolerance to multipole components in the cavity. In particular the dynamic aperture as a function of $b_{4}$ shows no significant drop 


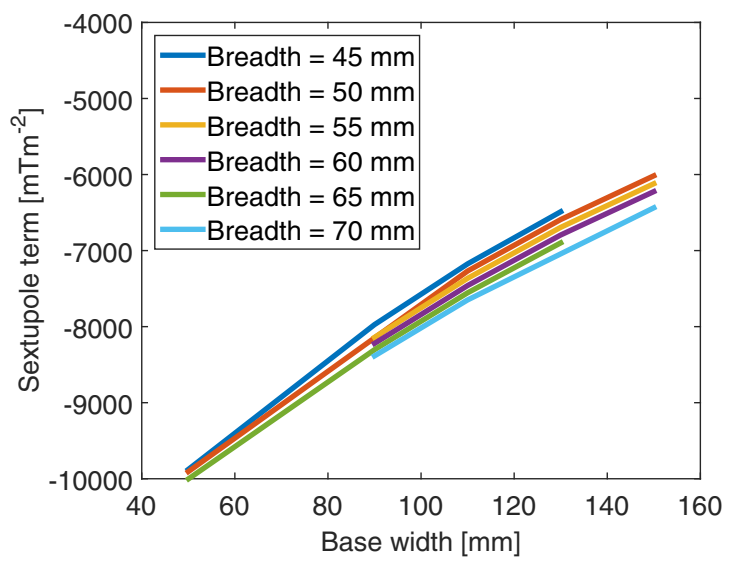

(a)

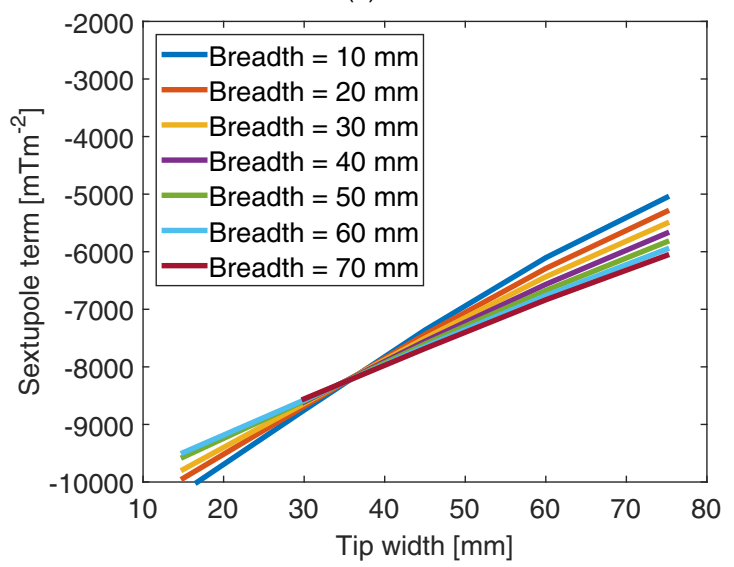

(b)

FIG. 13. Sextupole component vs rod width for the base ellipse (a) and tip ellipse (b).

up to values of the order of $1 \times 10^{5} \mathrm{mTm} / \mathrm{m}^{3}$. In addition the tune shift with amplitude is negligible for octupole components of this value. The complete assessment of the impact of a large $b_{5}$ (decapole) component is under study; however the tune shift is second order in the multipole strength, hence the dynamic aperture should be stable to values at least an order of magnitude in excess of the $b_{4}$ limit, so decapole strengths exceeding values of the order of $10^{6} \mathrm{mTm} / \mathrm{m}^{4}$ should be readily tolerable.

\section{A. Wing shaped rods}

It is however possible to modify the shape of the rods to manipulate the field; thus negating the sextupole term. In electron guns and kickers, focus electrodes are often used to make the fields uniform [19] and a similar method can be applied here. Figure 14 shows the sextupole component vs angle for the wing shaped rods; the sextupole terms are calculated from CST simulations using the method described in [20]. The rods have additional wings where the length and angle of the wings can be varied. For these simulations the base was fixed at $60 \mathrm{~mm}$. When the wing length exceeds the base length, no additional contribution

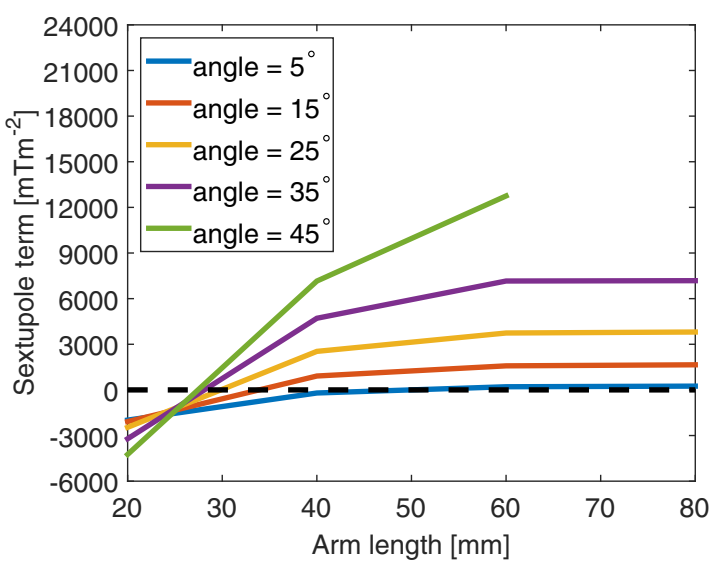

(a)

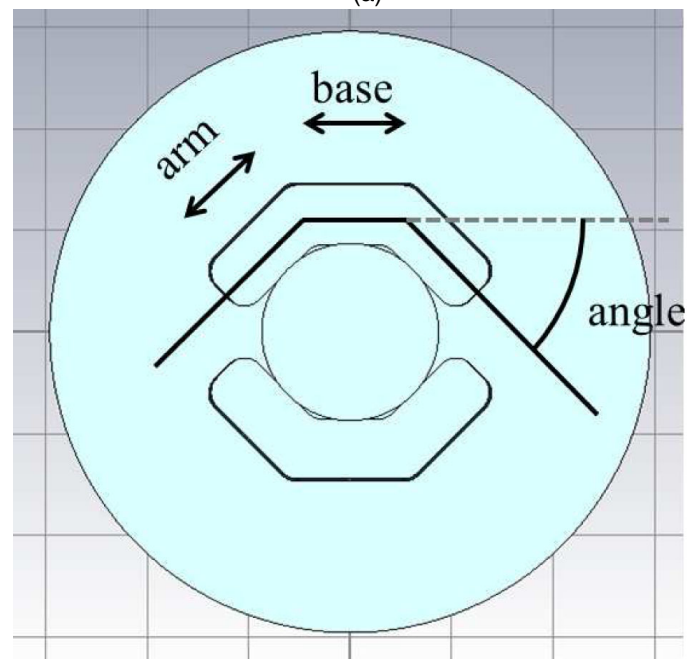

(b)

FIG. 14. (a) Sextupole component of wing shaped rods vs angle for a base width of $60 \mathrm{~mm}$ and (b) and the parametrization of the wing shapes.

to the sextupole field occurs and the sextupole field reaches a plateau. For a fixed arm length $>0$, the wing angle can be set to negate the sextupole field. A similar methodology could also be applied to the shaping of other transverse electric (TE) or transverse electromagnetic (TEM) crab cavities $[9,10]$.

\section{B. Kidney shaped rods}

The wings have been shown to correct the sextupole field, but the peak surface fields exceed the required tolerances due to the sharp corners and radii of the wings geometry; hence another rod shape is required to combine the low surface fields of the conical elliptical cavities with the low sextupole fields of the wings. The chosen rod design is a kidney shaped rod; this is drawn in CAD by designing a conical elliptical rod and removing the intersection with a "cutting ellipse" (Fig. 15). The kidney shaped rods are sufficiently smooth to reduce the surface fields to tolerable levels sufficiently flexible in design to 


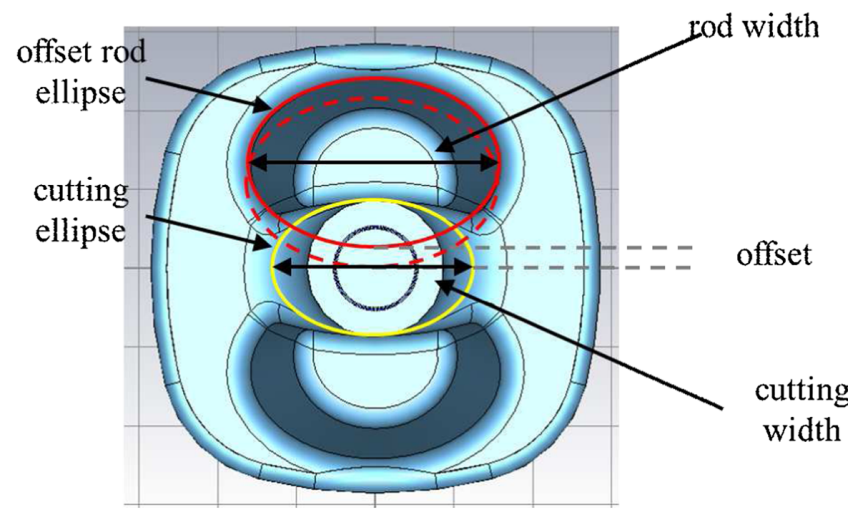

FIG. 15. Parametrization of the kidney shaped 4RCC.

tune the sextupole component. After removing the intersection of the cutting ellipse from the rod shape, a $20 \mathrm{~mm}$ rounding is applied to smooth the ellipse shape.

As can be seen in Fig. 16, the cutting ellipse width can be varied to negate the sextupole term for different rod widths. For a rod width of $110 \mathrm{~mm}$, to negate the sextupole component, the radius of the cutting ellipse needs to be smaller than the beam pipe radius; therefore the rods would encroach on the beam aperture, which is not acceptable for the LHC. Alternatively, the breadth of the cutting ellipse could be increased to negate the sextupole term; however this parameter was kept constant for these simulations. Figures 17 and 18 show that designing the rods to negate the sextupole term does not correspond to minimized peak surface electric and magnetic fields; thus a compromise is necessary. In these plots the tip width and breadth are $110 \mathrm{~mm}$ and $70 \mathrm{~mm}$, respectively, and the base width is varied, from this a solution is selected which satisfies the constraints on required transverse voltage and field uniformity.

\section{Final designs}

Two prototype cavities and a final cavity have been designed; one of the prototypes were manufactured by Niowave in niobium and the other by Lancaster University

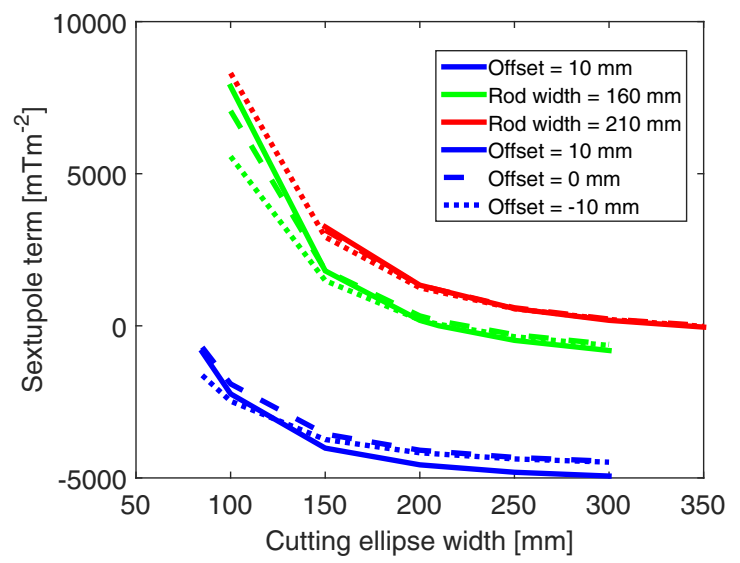

FIG. 16. Sextupole component vs cutting ellipse width for different rod widths and offsets.

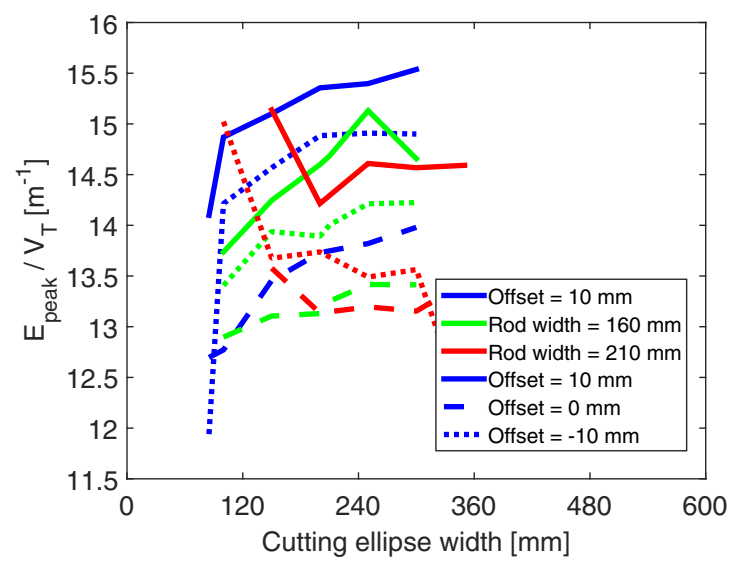

FIG. 17. $E_{\text {peak }} / V_{T}$ vs cutting ellipse width for different rod widths and offsets.

and the Cockcroft Institute in Aluminum. Table I summarizes the main parameters for three designs chosen for further study. The Niobium prototype was of design A (Fig. 19) which has narrower rods than the other designs to allow both halves of the prototype cavity to be milled from a single ingot of niobium to reduce the material cost. The Aluminum prototype cavity was of design $\mathrm{B}$ and had slightly wider rods in order to keep the $\mathrm{R} / \mathrm{Q}$ high for the dipole mode while keeping the sextupole component low; however this also meant having a high LOM R/Q. Although impedance calculations concluded that a low LOM R/Q was more important than a high dipole $\mathrm{R} / \mathrm{Q}$ hence a wide rod design $\mathrm{C}$ was also studied. The LOM R/Q values for these cavities are smaller than for the conical elliptical rods (Fig. 10) because the dimensions of the outer can are different.

A CAD drawings of the final cavity designs is shown in Fig. 20, looking along the beam axis. As can be seen, design $\mathrm{A}$ has the narrowest rods, and design $\mathrm{C}$ has the widest rods. As shown in previous sections, wider rods result in a higher $\mathrm{R} / \mathrm{Q}$ for both the crabbing mode and the LOM. The width of the cutting ellipse is the same for designs A and $\mathrm{B}$, and set to

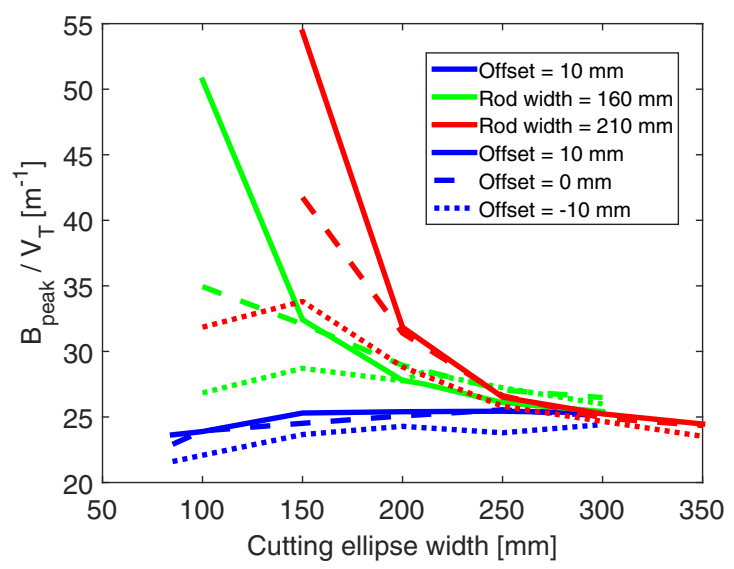

FIG. 18. $B_{\text {peak }} / V_{T}$ vs cutting ellipse width for different rod widths and offsets. 
TABLE I. A table of parameters for the final crab cavity designs.

\begin{tabular}{|c|c|c|c|}
\hline Parameter & Design A & Design $B$ & Design $\mathrm{C}$ \\
\hline Base width $[\mathrm{mm}]$ & 120 & 136 & 194 \\
\hline Base breadth [mm] & 74 & 74 & 96 \\
\hline Tip width $[\mathrm{mm}]$ & 100 & 110 & 175 \\
\hline Tip breadth $[\mathrm{mm}]$ & 70 & 70 & 96 \\
\hline Offset $[\mathrm{mm}]$ & 30 & 30 & 18 \\
\hline Cutting width [mm] & 84 & 84 & 265 \\
\hline Cutting breadth [mm] & 84 & 84 & 84 \\
\hline Cavity length [mm] & 500.7 & 500.7 & 524.8 \\
\hline Cavity diameter $[\mathrm{mm}]$ & 145 & 145 & 145 \\
\hline Longitudinal gap [mm] & 60 & 60 & 60 \\
\hline$E_{\text {peak }}$ at $V_{T}=3 \mathrm{MV}[\mathrm{MV} / \mathrm{m}]$ & 30.7 & 29.2 & 27.7 \\
\hline$B_{\text {peak }}$ at $V_{T}=3 \mathrm{MV}[\mathrm{mT}]$ & 54.9 & 58.9 & 63.9 \\
\hline Sextupole field $\left[\mathrm{mTm} / \mathrm{m}^{2}\right]$ & -898.5 & 474.33 & 321.57 \\
\hline Decapole field $\left[\times 10^{2} \mathrm{Tm} / \mathrm{m}^{4}\right]$ & -24.5 & -25.4 & -1.01 \\
\hline$R_{T} / Q[\Omega]$ & 538.11 & 504.72 & 325.00 \\
\hline LOM R/Q $[\Omega]$ & 130.85 & 124.28 & 102.97 \\
\hline LOM Frequency [MHz] & 374.60 & 375.27 & 371.41 \\
\hline HOM 1 Frequency $[\mathrm{MHz}]$ & 435.45 & 436 & 439.4 \\
\hline HOM 2 Frequency $[\mathrm{MHz}]$ & 449.72 & 452 & 456.19 \\
\hline HOM 3 Frequency [MHz] & 687.91 & 676 & 642.17 \\
\hline Geometric factor & 62.1 & 59.9 & 53 \\
\hline$\frac{G}{2} \frac{R}{Q}\left[\Omega^{2}\right]$ & 33416 & 30232 & 17225 \\
\hline
\end{tabular}

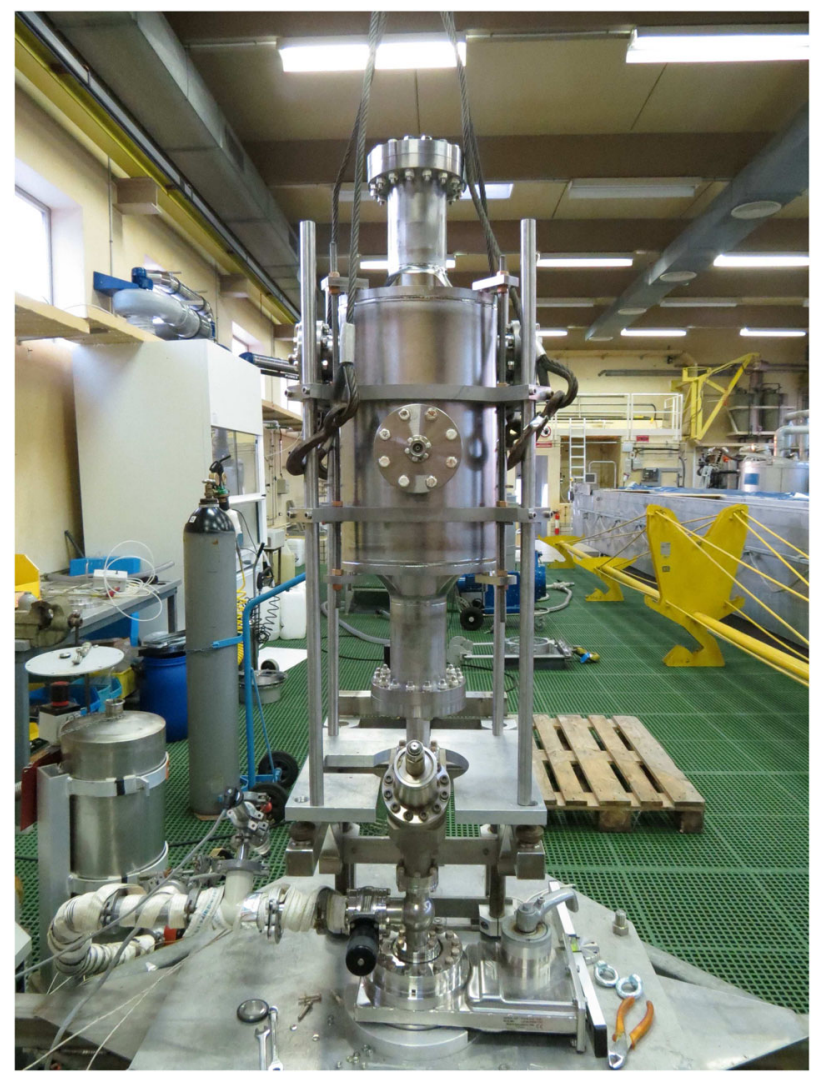

FIG. 19. The Niobium 4RCC prototype (Design A) dressed for cold rf testing.

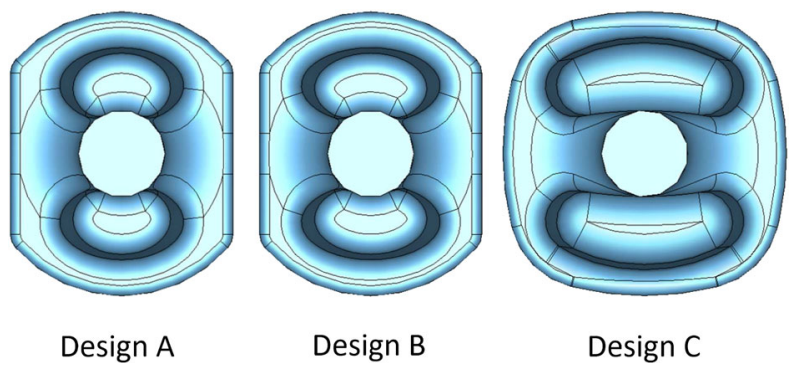

FIG. 20. CAD drawings of the final cavity designs viewed along the beam axis.

the beam pipe radius of $84 \mathrm{~mm}$, the cutting ellipse in design $\mathrm{C}$ is significantly wider, resulting in smaller multipole components as the dipole field is more uniform.

A high $\mathrm{R} / \mathrm{Q}$ is beneficial if the geometric factor, $G$, is also high as it minimizes losses, however if $\mathrm{R} / \mathrm{Q}$ is too high it can lead to issues with the low level RF (LLRF) stability [21]. The 4RCC has a very high R/Q compared to other TEM or TE crab cavities hence it is was felt that this could be traded off somewhat against other design parameters. Widening the rod has a strong affect on dipole R/Q, with wide rods having $40 \%$ less impedance. In addition the R/Q of the LOM was found to be strongly related to the R/Q of

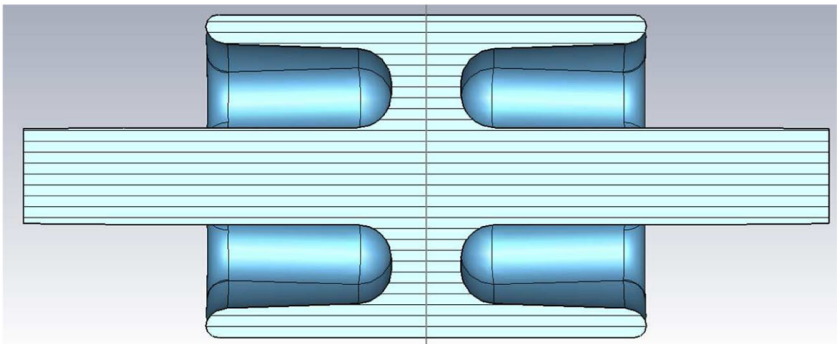

(a)

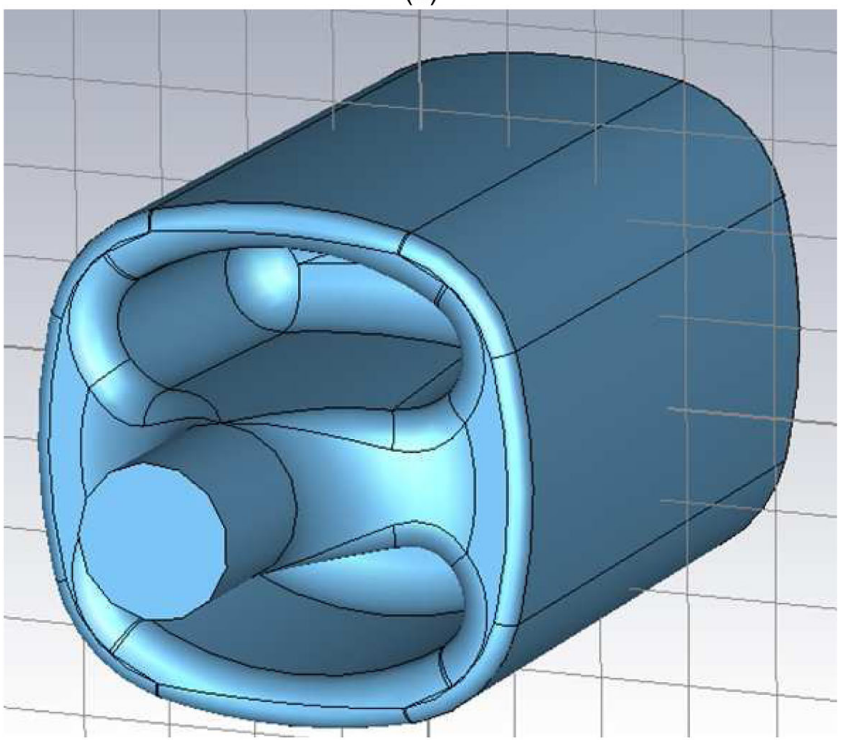

(b)

FIG. 21. Cross-sectional diagram of final cavity design C (a) and an isometric view (b). 
the dipole mode hence reducing the R/Q of the dipole mode has a similar effect on the LOM, with design C having 30\% less impedance than design A. The peak fields also increase as the rods get wider, with the peak magnetic field increasing from $55 \mathrm{mT}$ to $64 \mathrm{mT}$ from design A to design C. The frequencies of the first three higher order modes (HOMs) are only weakly dependent on the rod geometry and are mostly dependent on the rod length. The first two HOMs are the other two eigenmodes of the four quarter wave resonators, where the opposing rods have the same charge hence the shunt impedance of these modes are very low.

The final design was chosen as design $\mathrm{C}$, in order to keep the LOM impedance as low as possible at the expense of peak fields and dipole R/Q, and is shown in Fig. 21. Beam dynamics simulations have concluded that the sextupole field in this cavity design is tolerable and has a negligible effect on the dynamic aperture; the peak surface fields are sufficiently low as to allow for stable operation without significant ohmic heating and quenching. The lower dipole R/Q of this design would result in a higher cryogenic load but it was still relatively high. The low LOM R/Q of this design will also result in a low longitudinal impedance experienced by the passing bunches; longitudinal impedance is a major concern for HL-LHC and must therefore be minimized.

\section{PERTURBATION MEASUREMENTS}

In order to see if it was possible to verify the reduction in the sextupole term due to the kidney shaped rods an aluminum prototype was manufactured (Fig. 22). The geometry for the aluminum cavity (design B) was chosen at an early stage in the design before the LOM impedance limits were known, and design B was chosen, although this was not the design for the final cavity. However an effective method of accurately measuring the sextupole rf field component in a cavity is not currently known. In magnets a rotating coil can be used [22] but this method becomes complex at rf frequencies hence another method must be utilized. The multipole components of the rf field in the

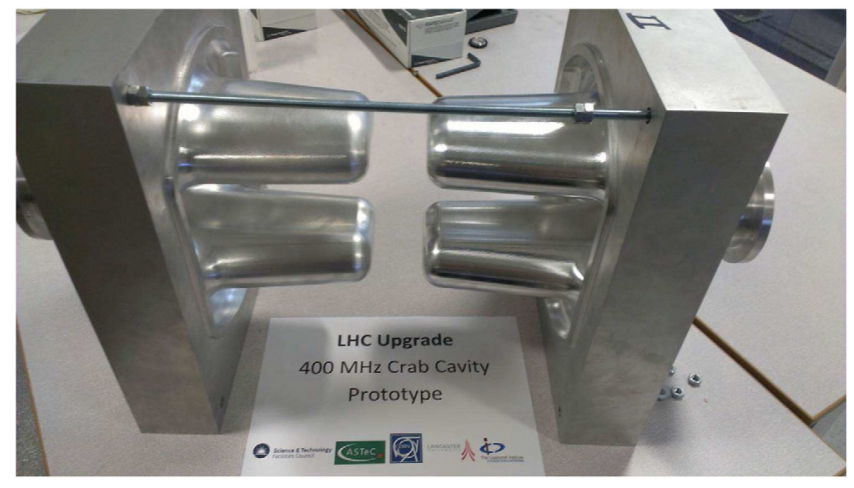

FIG. 22. The Aluminum cavity without the outer can, showing the inner rods. cavity can be decomposed by observing the variation of the longitudinal electric field either radially or azimuthally. The radial variation is simpler to measure hence we have selected to initially investigate this method to ascertain the accuracy of such a measurement and hence if it can be used to measure the sextupole component in crab cavities.

In order to measure the longitudinal electric field, a resonant perturbation technique, commonly known as a bead pull, was utilized to measure the field amplitude at different radial positions along the cavity [23]. The bead pull method uses a metallic or dielectric perturbing object (normally a circular bead or cylindrical needle) threaded on a thin dielectric thread (in this case Kevlar). As the perturbing object is moved through the cavity a local change in the energy density causes a frequency or phase shift, proportional to the value of the local field squared, which can be measured by a network analyzer. A circular bead will affect all field directions equally whereas a needle is more sensitive to field parallel to its major axis; hence a longitudinal needle is required to measure the longitudinal electric field. The frequency shift, $\Delta f_{r}$, due to a metal needle is proportional to the sum of the squares of the electric and magnetic fields around the perturbing object multiplied by a polarizability factor, $\alpha_{i}$, for each field component. $f_{0}$ is the unperturbed frequency and $W$ is the energy stored in the cavity. Assuming the perturbation is small the frequency shift, which can be converted into a phase shift if the Q is known, due to a metallic needle has the following form:

$\frac{\Delta f_{r}}{f_{0}}=\frac{\varepsilon_{0}\left(\alpha_{E_{z}} E_{z}^{2}+\alpha_{E_{T}} E_{T}^{2}\right)+\mu_{0}\left(\alpha_{M_{z}} H_{z}^{2}+\alpha_{M_{T}} H_{T}^{2}\right)}{W}$.

The electric $\left(\alpha_{E}\right)$ and magnetic $\left(\alpha_{M}\right)$ polarizability of a solid metallic elliptical needle is calculated below where $\beta=b / a$ with $a$ being the radius of the ellipsoid's major longitudinal axis and $b$ being the radius of the ellipsoid's minor transverse axis [23]. For a hollow cylindrical metal needle we can approximate the geometry as that of a solid elliptical needle with the radius as the minor axis radii $b$ and the length as the major axis diameter $2 a$

$$
\begin{aligned}
& \alpha_{E_{z}}=\frac{-\frac{2}{3} \pi a^{3}\left(1-\beta^{2}\right)^{\frac{3}{2}}}{\ln \frac{1+\left(1-\beta^{2}\right)^{\frac{1}{2}}}{1-\left(1-\beta^{2}\right)^{\frac{1}{2}}}-2\left(1-\beta^{2}\right)^{\frac{1}{2}}} \\
& \alpha_{M_{z}}=\frac{-\frac{2}{3} \pi a^{3}\left(1-\beta^{2}\right)^{\frac{3}{2}}}{\ln \frac{1+\left(1-\beta^{2}\right)^{\frac{1}{2}}}{1-\left(1-\beta^{2}\right)^{\frac{1}{2}}}-\frac{2\left(1-\beta^{2}\right)^{\frac{1}{2}}}{\beta^{2}}} \\
& \alpha_{E_{T}}=\frac{-\frac{4}{3} \pi a^{3}\left(1-\beta^{2}\right)^{\frac{3}{2}}}{\ln \frac{1+\left(1-\beta^{2}\right)^{\frac{1}{2}}}{1-\left(1-\beta^{2}\right)^{\frac{1}{2}}}-\frac{2\left(1-\beta^{2}\right)^{\frac{1}{2}}}{\beta^{2}}} \\
& \alpha_{M_{T}}=\frac{-\frac{2}{3} \pi a^{3}\left(1-\beta^{2}\right)^{\frac{3}{2}}}{\ln \frac{1+\left(1-\beta^{2}\right)^{\frac{1}{2}}}{1-\left(1-\beta^{2}\right)^{\frac{1}{2}}}-\frac{2\left(1-\beta^{2}\right)^{\frac{1}{2}}\left(1-2 \beta^{2}\right)}{\beta^{2}}} .
\end{aligned}
$$


As we are measuring a dipolar electric field close to the axis, where the field is close to zero, we need a needle as a perturbing object. We have chosen to use a metallic needle which produces a larger perturbation than a dielectric needle, however this also results in a perturbation due to the transverse magnetic field which needs to be taken into account. A needle can be used to measure the longitudinal electric field with error bars equal to the perturbation from the other field components. A cylindrical needle of $30 \mathrm{~mm}$ length and $0.8 \mathrm{~mm}$ radius was used to measure the longitudinal electric field $\left(E_{z}\right)$ of the 4RCC. The error due to the other field components for this needle can be calculated using the fields from a CST simulation. The transverse magnetic field provides a perturbation that is $16 \%$ of $E_{z}$ and the transverse electric field provides a perturbation that is $19 \%$ of $E_{z}$, at an offset of $10 \mathrm{~mm}$ in the cavity. The error due to these components can be calibrated out if those fields are known. We can make the assumptions that the transverse electric and magnetic fields are approximately constant across the aperture (a CST simulation shows this to be a reasonable approximation, Fig. 23) and that the longitudinal magnetic field component is zero. From this a measurement of the frequency shift with the needle at the center of the cavity, where $E_{z}=0$, can measure the perturbation from the transverse field components only; which we will refer to as $\Delta f_{T} / f_{0}$. The measurement can then be subtracted from the measurement at larger radial offsets to leave only the longitudinal term. There is also an uncertainty in the measurement due to the finite length of the needle, as the field amplitude will vary along it and we use a hollow cylindrical needle rather than a solid elliptical one. However both these effects can be calibrated out with the use of CST simulations and will likely be identical at different offsets and hence will not affect relative measurements of the voltage at different offsets.

In addition to the errors due to the other field components and the finite size of the needle there is also random electrical noise on the measurement. As we require the square root of the frequency/phase shift along the cavity length to get the field it is necessary to take the magnitude of the

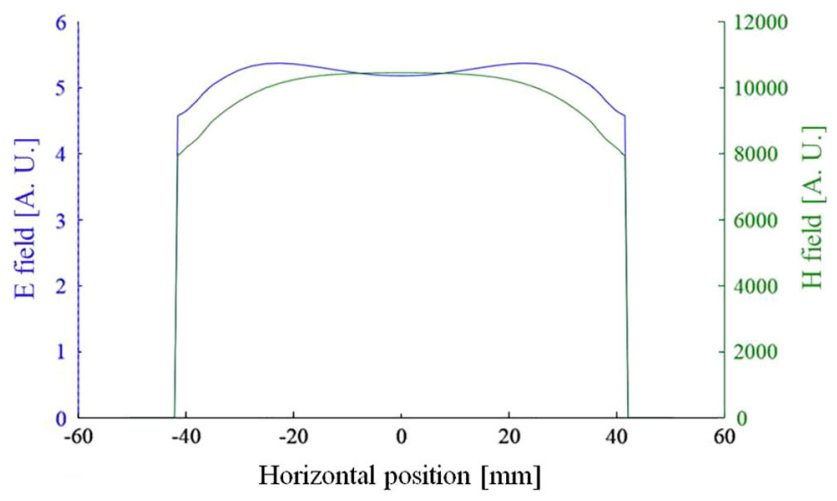

FIG. 23. Transverse E field (blue) and H field (green) vs horizontal position in arbitrary units (A. U.).
TABLE II. A table of design parameters of the control elliptical conical cavity design [24].

\begin{tabular}{lc}
\hline \hline Parameter & Value \\
\hline Rod gap [mm] & 60 \\
Length [mm] & 402 \\
Beam pipe radius [mm] & 42 \\
Outer radius [mm] & 145 \\
Beam pipe rounding [mm] & 30 \\
Rod base rounding [mm] & 20 \\
Tip rounding [mm] & 20 \\
Tip width [mm] & 65 \\
Tip breadth [mm] & 70 \\
Mid width [mm] & 67.5 \\
Mid breadth [mm] & 90 \\
Base width [mm] & 70 \\
Base breadth [mm] & 110 \\
$\mathrm{E}_{\max }$ at $3 \mathrm{MV} \mathrm{[MV/m]}$ & 37.0 \\
$\mathrm{~B}_{\max }$ at $3 \mathrm{MV}[\mathrm{mT}]$ & 66.2 \\
\hline \hline
\end{tabular}

measurement; thus the noise does not cancel out over several measurements or along the length. When the magnitude of the signal due to the noise is integrated along the cavity length to get a voltage we find that it adds a finite dc offset to the voltage measurements. Several measurements of the needle moving through the cavity center, where $E_{z}=0$, were performed. By subtracting the phase from two identical runs and integrating we can get a measurement of the voltage error due to the noise. By repeating this over several measurements we found this noise voltage to be fairly constant over the course of an hour and hence can be calibrated out of measurements performed soon after.

In order to differentiate between a cavity with a high and low sextupole component the measurements are compared to CST simulations of an elliptical conical cavity as well.

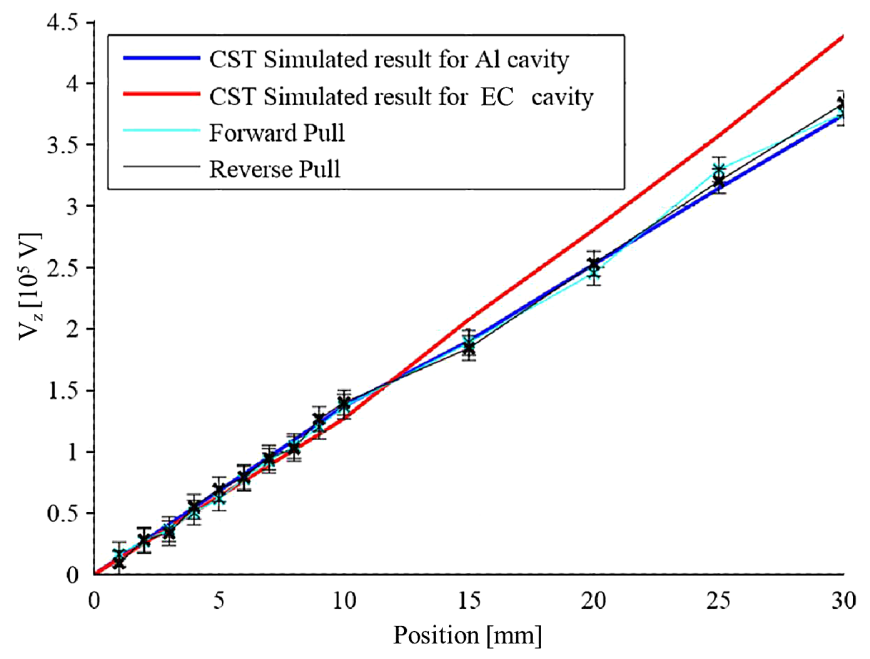

FIG. 24. Comparison of bead pull measurements and CST simulations of relative voltage with predicted longitudinal voltage against bead or beam offset. "EC" represents the elliptical conical cavity. 
TABLE III. A table of the multipolar components of the elliptical conical and Aluminum cavity designs, normalized to a transverse voltage of $10 \mathrm{MV}$.

\begin{tabular}{lcc}
\hline \hline Multipole component & Elliptical conical & Aluminum \\
\hline Dipole $[\mathrm{mTm}]$ & 33.3 & 33.3 \\
Sextupole $\left[\mathrm{mTm} / \mathrm{m}^{2}\right]$ & -6383.8 & 474.3 \\
Decapole $\left[\mathrm{mTm} / \mathrm{m}^{4}\right]$ & $8.49 \times 10^{5}$ & $-2.54 \times 10^{6}$ \\
\hline \hline
\end{tabular}

The design parameters for the elliptical conical cavity are summarized in Table II [24]. The measured signals are scaled to the CST simulation results to account for the uncertainty in the polarizability of the needle due to the use of a cylindrical rather than elliptical needle. The measurements show that the longitudinal voltage is roughly linearly dependent on the transverse offset in the cavity as expected; hence producing a uniform deflecting voltage in good agreement with the simulation results.

The measurement results are shown in Fig. 24 and compared to simulation results from CST Microwave Studio [13]. It can be seen that the CST simulation results for the longitudinal voltage for cavity design B do not vary greatly for small offsets as the sextupole component is proportional to $r^{3}$ whereas the dipole varies with $r$. Thus a sufficiently large transverse offset is required to observe a statistically significant change in $V_{z}$ due to the sextupole component; the dipole, sextupole, and decapole terms for the elliptical conical and Aluminum cavities are shown in Table III. At an offset of $30 \mathrm{~mm}$ the bead pull measurement of transverse voltage variation is accurate to $\sim 2 \%$; resulting in a minimum sextupole amplitude sensitivity of $\sim 60 \mathrm{mTm} / \mathrm{m}^{2}$ with a dipole transverse voltage of $10 \mathrm{MV}$. For the Aluminum crab cavity, at $30 \mathrm{~mm}$ transverse offset, the decapole field is approximately 5 times larger than the sextupole field; thus making the sextupole component difficult to measure. If the sextupole field were measured at a transverse offset of $4 \mathrm{~mm}$, the field contribution from the sextupole term would be 10 times larger than the contribution from the decapole term. However at this offset, the signal to noise ratio of the measurement would be approximately 1 ; thus preventing any statistically significant measurements of the sextupole term. By comparison, for the elliptical conical crab cavity, the decapole field is approximately $10 \%$ of the amplitude of the sextupole field at $30 \mathrm{~mm}$ transverse offset. Further work will investigate if better sensitivity can be obtained by measuring the azimuthal variation in longitudinal voltage rather than the radial variation.

\section{LOM AND HOM COUPLERS}

One issue with 4RCCs is the LOM. Most HOM couplers utilize high pass filters along with bandstop filters to ensure that the operating mode has a high Q, but with a LOM in the cavity this would also not be damped due to the high pass filter. This means that a 4RCC also needs an additional
LOM coupler. If the LOM and the operating mode of the cavity can be separated then the coupler could utilize a low pass filter and a bandstop filter, however for LHC the LOM is very close to the dipole mode at $\sim 370 \mathrm{MHz}$. Thus only a bandstop can be utilized without using a complicated multielement low-pass filter, and care must be taken in the couplers' circuit to ensure strong damping of the LOM $(\mathrm{Q} \sim 500)$ and close to no damping of the crabbing mode $\left(\mathrm{Q} \sim 10^{9}\right)$. However just using a bandstop would require very tight mechanical tolerances so this alone is not allowable. Instead we use the different field patterns of the crabbing mode and the LOM to place the coupler in a location with high LOM fields but no fields in the crabbing mode. For horizontal crabbing there is no field in the vertical plane hence a LOM coupler can be placed there, with high electric fields in the LOM near the end of the rods and a high magnetic field near the base.

It has been determined from CST simulations [13] that a magnetic coupler provided significantly more damping than electric coupling so the coupler design chosen is a loop to couple to the high magnetic field in the LOM at the base of the rods. The bandstop filter is created by adding an annular section which is capacitively coupled to the outer conductor and connected to the inner conductor via a stub. The inductance of the stub and the capacitance of the annular ring provide a bandstop at $400 \mathrm{MHz}$. Two support rods were added to keep the coupler aligned to the field null of the crabbing mode, these were positioned $50 \mathrm{~mm}$ away from the loop in order to fit a flange between the support and the cavity making the whole system demountable for tuning and cleaning. As the coupler has low fields in the crabbing mode there is no issue related to heating of the gasket by the crabbing mode. The pick-up is also coupled capacitively to make this section also removable for ease of assembly. The length of the inner conductor between the filter and the tip of the loop, and the length between the filter and the feed-through are both chosen to increase coupling at $370 \mathrm{MHz}$ by creating two resonances. The final coupler is shown in Fig. 25 and achieves an external Q of
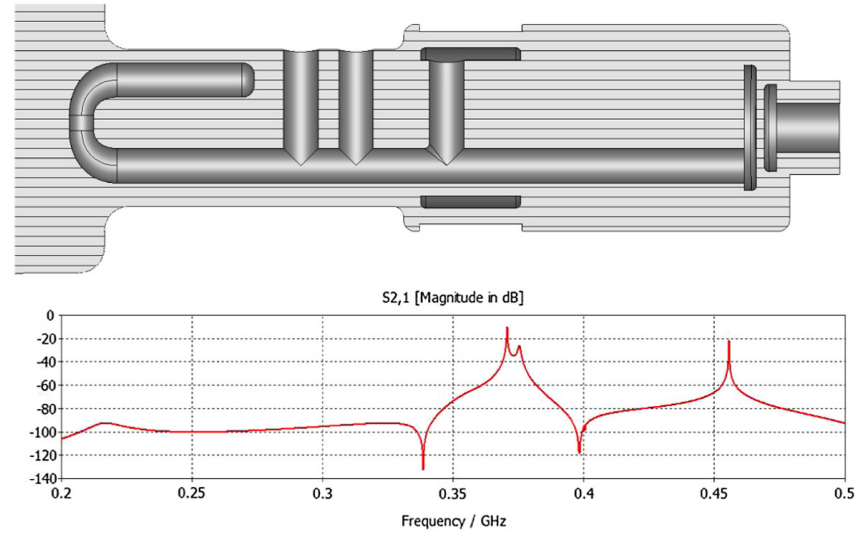

FIG. 25. LOM coupler geometry (top) and transmission spectrum (bottom). 
451 for the LOM when coupled to the cavity. This coupler was also found to damp all monopole modes up to $2 \mathrm{GHz}$.

As this coupler is placed in a field null of the crabbing mode the rf losses on this coupler are very low. The heat load due to the LOM itself is less than $100 \mathrm{~mW}$.

As with all SRF cavities HOM couplers are needed to damp all the monopole and dipole modes above $400 \mathrm{MHz}$. One complication is the dipole mode at $440 \mathrm{MHz}$ which has longitudinally opposite rods with the same polarity. This mode is close to the operating frequency of the cavity and hence any high pass filter is likely to attenuate this frequency strongly. To aid in the damping of this mode it was chosen to have three HOM couplers, one for vertical modes, one for horizontal modes and one especially for the $440 \mathrm{MHz}$ mode. The $440 \mathrm{MHz}$ mode coupler is a simple probe with a notch filter to reject the crabbing mode. This coupler would be placed at the longitudinal center of the cavity so that it does not couple to the crabbing mode, hence allowing the coupler to safely operate without a high pass filter, but does couple to the $440 \mathrm{MHz}$ mode, a notch filter is added only to protect against coupler misalignment. The other option would be to damp the $440 \mathrm{MHz}$ mode with the horizontal HOM couplers but the coupler design becomes very complex.

The coupler for damping vertical HOMs is a probe type coupler and the coupler for horizontal HOMs is a loop type coupler at the base. Both couplers are mounted on the bottom of the cavity and have right angled bends so the cables run longitudinally due to space limitations. Both couplers have a notch filter created by an annular ring connected to the inner conductor near the tip of the coupler (shown in Fig. 26). This shields the flange/gasket from the crabbing mode's magnetic fields allowing the couplers to be demountable. Without the notch filter there would be excessive heating on the copper gasket. This notch filter is used on all four couplers (including the LOM). The HOM couplers incorporate a high-pass filter in the right angled bend in case the notch filter's center frequency is moved during assembly or cool-down. This coupler has good

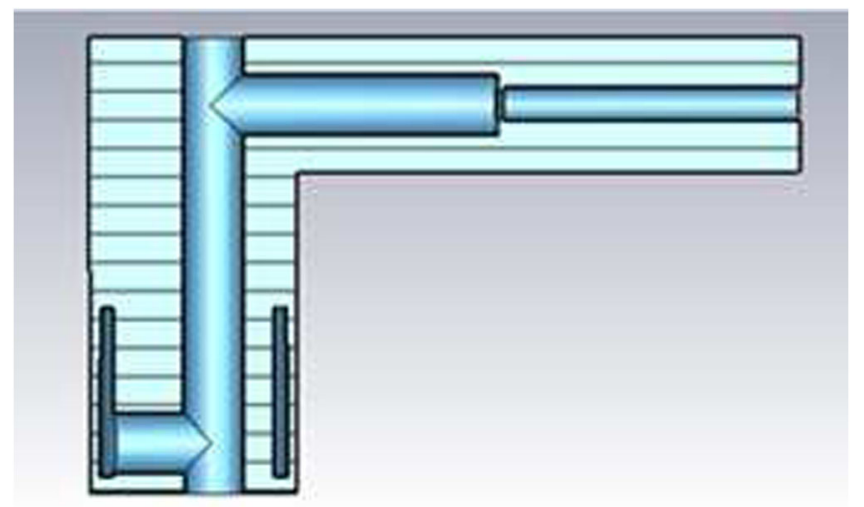

FIG. 26. Filter design for the vertical and horizontal HOM couplers.

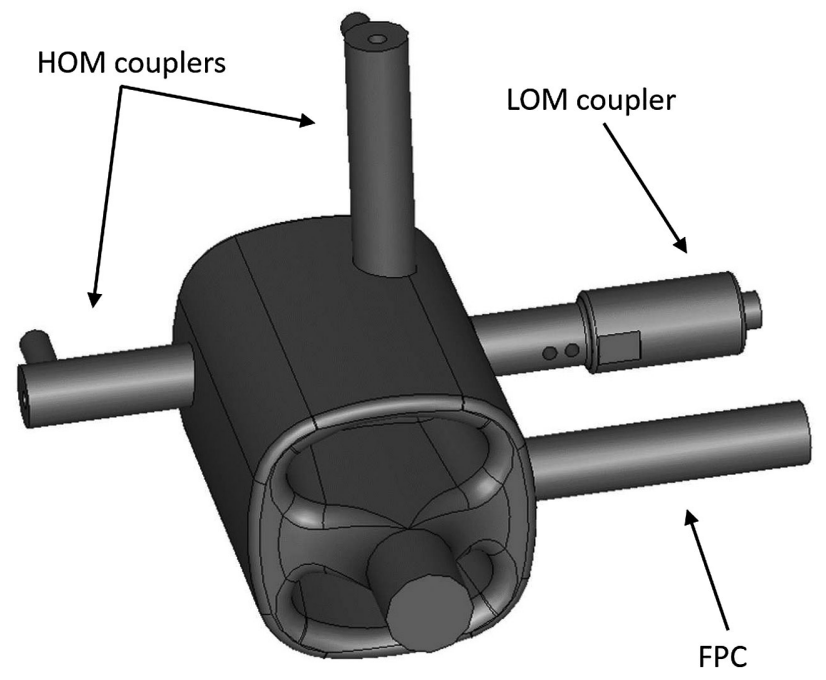

FIG. 27. A CAD drawing of the $4 \mathrm{RCC}$ with the HOM and LOM couplers as well as the fundamental power coupler (FPC).

rejection of frequencies up to around $550 \mathrm{MHz}$, and has a broad passband above this frequency up to $2 \mathrm{GHz}$ allowing good damping of all HOMs. The passband has three peaks which were adjusted to have maximum damping at the frequencies of three HOMs that required additional damping after assessing the impedance of the cavity with an initial design. A modified version of this HOM coupler design has also been proposed for the double-quarter wave crab cavity for LHC. A CAD drawing of the $4 \mathrm{RCC}$ with the $\mathrm{HOM}$ and LOM couplers as well as the fundamental power coupler (FPC) is shown in Fig. 27.

A simulation of cavity design $\mathrm{C}$ with all HOM/LOM couplers was performed in CST Microwave studio. The combination of all four couplers is found to damp all modes up to $2 \mathrm{GHz}$ to the longitudinal and transverse impedance limits of $2.4 \mathrm{M} \Omega$ and $1.5 \mathrm{M} \Omega / \mathrm{m}$ total over 16 cavities per beam as shown in Fig. 28 [25]. The requirement for the 4 couplers could be relaxed if a higher impedance could be tolerated after detailed impedance modeling with a realistic spectrum. The impedance requirement for HL-LHC has indeed been relaxed for the other crab cavity concepts in this manner and the impedance of the 4RCC presented here is now nearly an order of magnitude lower than the current

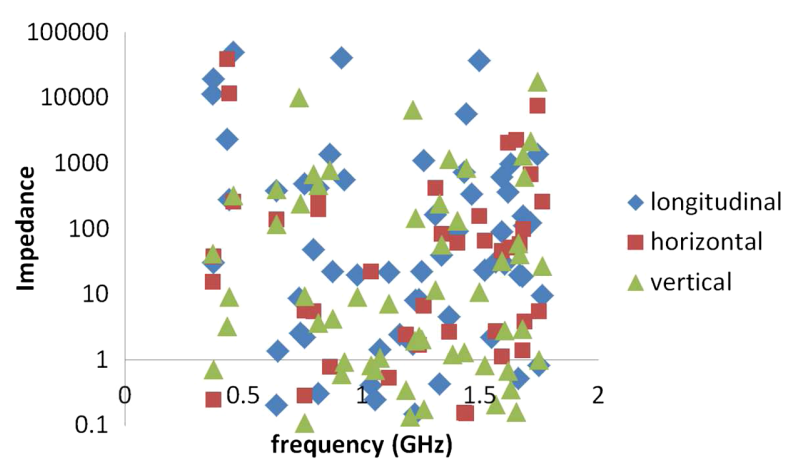

FIG. 28. Modal impedance for the final 4RCC design. 
modified specification. However in order to achieve this four $\mathrm{HOM} / \mathrm{LOM}$ couplers are required, however one of these is a simple probe with a notch and the geometry of the filters is identical for both HOMs simplifying the manufacture. The current modified impedance specifications could potentially be met with less HOM couplers.

\section{TESTING}

A prototype of cavity design A was manufactured in Niobium in 2012 by Niowave in the US and was the first of the 4RCC designs to be manufactured and tested. The rods and base plates were machined from a single ingot of Niobium; avoiding the need for complicated electron beam welding around the rods. However, during manufacture one of the rods did not meet design specifications and so needed to be removed and a replacement rod welded in its place.

Between 2013 and 2015, the prototype 4RCC underwent 3 cold tests at the SRF vertical test station in SM18 at CERN. During the tests, the cavity was monitored with a range of sensors distributed around the cavity. This included oscillating superleak transducers (OSTs) to detect quenches by measuring 2nd sound oscillations in the superfluid liquid Helium [26], 18 contact Cernox temperature sensors [27] placed on the inside of the rods and around the outer can, helium level gauges, cavity and helium bath pressure gauges and 3 single-axis magnetic flux probes to measure residual ambient magnetic field $[28,29]$.

During cold testing, the cavity is cooled from $300 \mathrm{~K}$ to $15 \mathrm{~K}$ at a rate of $15 \mathrm{~K} / \mathrm{min}$. Niobium has a superconducting transition temperature of $9.2 \mathrm{~K}$, where the thermal conductivity suddenly drops. At $15 \mathrm{~K}$, the cavity is allowed to reach thermal equilibrium and then the cavity is cooled at a rate of $0.9 \mathrm{~K} / \mathrm{min}$; this limits the spatial thermal gradient to less than $2 \mathrm{~K}$ over the entire cavity; hence avoiding thermal electric currents. Cooling at a low rate allowed the cavity losses to be monitored as a function of temperature to be monitored throughout. Due to the geometry of the cavity and its vertical orientation in the cryostat, there was the potential for flux trapping at the superconducting transition at the tips of the bottom rods, but this was not seen as an issue because the rf magnetic field should be close to zero at this location.

The first cold test on the 4RCC took place in March 2013 , after a $120^{\circ} \mathrm{C} 24$-hour bake out, but before the final light buffered chemical polish (BCP). The cavity achieved a transverse voltage of $1.6 \mathrm{MV}$ with a low field Q-factor of $1 \times 10^{9}$; equivalent to $45 \mathrm{n} \Omega$ of residual resistance. Although the transverse voltage was significantly lower than the $3 \mathrm{MV}$ design value, this was the first compact crab cavity to exceed $1 \mathrm{MV}$ transverse voltage. It was discovered that steel components in the vacuum system had resulted in a residual magnetic field of approximately $10 \mu \mathrm{T}$; which likely caused the large residual resistance in the cavity and hence the drop in transverse voltage. The cavity also had problems sustaining a high vacuum during this test and a vacuum pressure of approximately $1 \times 10^{-7}$ mbar at $2 \mathrm{~K}$ was measured.

After this initial test, the cavities $\mathrm{Nb}$ - $\mathrm{Ti}$ flanges were inspected to understand the cause of the high vacuum pressure, and significant burring was found on the knifeedges. The cavity flanges were then remachined after which the cavity underwent a BCP with an average removal thickness of $20 \mu \mathrm{m}$, followed by a high-pressure rinsing with 100 bar ultrapure water in order to remove any contaminants from the surface. The high pressure rinse was comprised of 6 cycles, with a two-jet nozzle traveling through the cavity at $0.5 \mathrm{~mm} / \mathrm{s}$ and rotating at an angular speed of $3 \mathrm{rpm}$ to ensure the whole inner surface of the cavity was rinsed. The cavity was accessed for rinsing from the beampipes, and as such there was no direct line of sight from the jet to the back of the rods, hence leaving the possibility of remaining contamination at these locations. After the rinsing, the cavity was dried in an ISO4 clean room environment for 12 hours with a laminar air flow in the direction of the beam axis.

The cavity was retested in December 2013, under the same conditions as the first test and achieved a transverse voltage of $3.3 \mathrm{MV}, 10 \%$ higher than the design value of $3 \mathrm{MV}$; however the Q-factor was measured to be $1.1 \times 10^{9}$. $3.3 \mathrm{MV}$ is equivalent to a peak electric field of $35 \mathrm{MV} / \mathrm{m}$ and a peak magnetic flux density of $65 \mathrm{mT}$ which is lower than the expected maximum limiting values, implying higher transverse fields could potentially be achieved in future testing. Negligible radiation was produced during this test, hence field emission was not an issue, although the thermal sensors and the 2 nd sound quench detection system [26] suggest that the cavity was undergoing a thermal quench near the weld on the side of the replacement rod. As there is a small magnetic field component perpendicular to the weld in this region, it is likely that this led to heating of the weld region leading to a thermal quench. As little heating was observed on the other rods it suggests that a base plate made from a single piece would likely reach a higher transverse gradient.

After the second cold test, the low Q-factor (or large residual resistance) was investigated. It was revealed that the input and pickup probes where manufactured from stainless steel rather than copper. As the probes penetrated into the cavity fields this led to rf losses, a proportion of which appeared as Ohmic losses in the cavity rather than cable losses. CST simulations verified that the error in the measured residual resistance of the cavity due to the stainless steel probes were approximately $22 \mathrm{n} \Omega$ instead of $9 \mathrm{n} \Omega$ for copper probes.

A third test was undertaken in August 2015 with new copper probes, however the high pressure rinse system was later found to have a contamination issue, thus the cavity could not operate at high gradient due to contaminants introduced in this process causing field emission at high field. Thus the maximum transverse voltage measured was only 2.5 MV, although the Q-factor had increased to the 


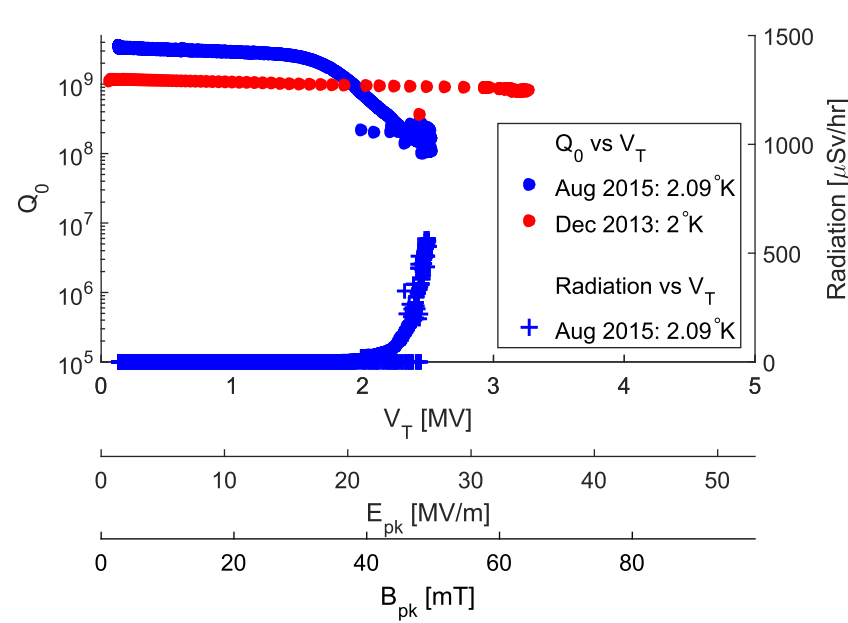

FIG. 29. Q verses transverse voltage measurements, and radiation monitor readings of the Niowave $4 \mathrm{RCC}$ tests.

expected value of $3.5 \times 10^{9}$, equivalent to a residual resistance of $15 \mathrm{n} \Omega$. Of this residual resistance, $9 \mathrm{n} \Omega$ was attributed to the copper probes from CST simulations and an estimated $6 \mathrm{n} \Omega$ attributed to the Ohmic losses in the cavity. This corresponds to a power dissipation of approximately $4.5 \mathrm{~W}$ from the cavity during normal operation, which compares favorably with the cryogenic system; which is designed for a maximum cavity heat load of approximately $6 \mathrm{~W}[30]$.

The cavity will be subjected to further testing in early 2017 after improvements to the high pressure rinse system at CERN. The results of the second and third cavity cold tests are presented in Fig. 29.

\section{CONCLUSIONS}

In this paper we present a novel design of a transversely compact crab cavity. Four quarter-wave rods, aligned longitudinally, provide a deflecting field where transversely opposing rods have opposite polarity. Important cavity parameters, such as peak surface fields, lower order mode impedance and multipolar components in the cavity are all governed by the shape of the rods in the cavity. Different rods shapes are investigated and show that conical elliptical rods can be used to minimize the peak surfaces fields in the cavity, but they produce large sextupolar fields. The sextupole field from the crab cavity changes the energy dependent tune spread, or chromaticity, of the bunches and introduces other chromatic and geometric aberrations which reduce the dynamic aperture of the LHC.

We show that the introduction of focus electrodes can be used to reduce or even eliminate the sextupole component in the cavity. We present this technique as a method to improve field uniformity in crabbing and deflecting cavities; the shape of the rods or focus electrodes can always be optimized to completely negate the net sextupole contribution of the cavity, however this leads to high surface fields. A kidney shaped rod design has been developed as a compromise between the conical elliptical rods and wing shaped rods to produce a cavity with relatively low peak surface fields and a tolerable sextupole component. The final cavity design has $E_{\text {peak }}=27.7 \mathrm{MV} / \mathrm{m}, B_{\text {peak }}=63.9 \mathrm{mT}$, and the amplitude of the sextupole field component is $321.57 \mathrm{mTm} / \mathrm{m}^{2}$ at $10 \mathrm{MV}$ deflecting voltage. Beam dynamics studies have been undertaken and suggest that the 4RCC design presented in this paper does not cause a significant detriment to the dynamic aperture of the LHC [15].

A prototype cavity was manufactured from aluminum to verify simulation results from CST Microwave Studio. Bead pull measurements were performed at a range of offsets and estimated errors were calculated from repeated measurements. With the given errors the measurement can only distinguish between the field profile of the elliptical conical and kidney shaped rods at an offset of $30 \mathrm{~mm}$. However, at this offset the amplitude of the decapole field for the kidney shaped, aluminum crab cavity is approximately 5 times larger than the sextupole field making it difficult to measure the exact sextupole amplitude. Future work will investigate measuring the change in voltage by moving the bead in a circle around the beam-axis.

Suitable couplers that damp the cavities LOM and HOM are presented. The LOM at $\sim 370 \mathrm{MHz}$ is damped (Q 500) while rejecting the operating mode $\left(\mathrm{Q} \sim 10^{9}\right)$. Similarly the HOMs are damped by HOM couplers that damp all remaining HOMs up to $2 \mathrm{GHz}$. The combination of all 4 couplers ensures that the impedance limits of $2.4 \mathrm{M} \Omega$ and $1.5 \mathrm{M} \Omega / \mathrm{m}$ are met for the full LHC layout with 16 cavities.

A prototype cavity was manufactured in niobium and repeatedly tested in SM18 at CERN. Initially the cavity was able to achieve a transverse voltage of 3.3 MV. However the Q was lower than desired at $1.1 \times 10^{9}$. This low value was found to be due to the couplers being manufactured from stainless steel rather than copper. The cavity was then reprocessed and achieved a $\mathrm{Q}$ of $3.5 \times 10^{9}$ in subsequent testing. However due to contamination issues with the high pressure rinse system the cavity was only able to achieve a gradient of 2.5 MV in this test. Further tests are planned with an improved high pressure rinse system during 2017.

\section{ACKNOWLEDGMENTS}

The work undertaken in this paper was funded by the Cockcroft Institute Core Grant (STFC grant reference ST/ G008248/1), HL-LHC-UK (supported by STFC grant reference ST/N001575/1 and by CERN) and HiLumi FP7 (grant agreement 284404).

[1] F. Zimmermann and U. Dorna, 2nd CARE-HHH-APD Workshop on Scenarios for the LHC Luminosity Upgrade, Arcidosso, Italy, pp. 55-73 (CERN, Geneva, 2005), LHC-LUMI-05.

[2] R. B. Palmer, SLAC Report No. SLAC-PUB-4707, 1988. 
[3] W. Bauer, A. Citron, G. Dammertz, H. Diepers, H. C. Eschelbacher, H. Lengeler, H. Miller, and E. Rathgeber, RF-tests on deflecting cavities for a superconductinig particle separator, IEEE Trans. Nucl. Sci. 20, 59 (1973).

[4] K. Hosoyama et al., in Proceedings of the 11th European Particle Accelerator Conference, Genoa, 2008 (EPS-AG, Genoa, Italy, 2008), p. 2927, THXM02.

[5] R. Calaga, in Chamonix 2012 Workshop on LHC Performance (CERN, Geneva, 2012).

[6] C. Leeman and H. Wang et al., Proceedings of LINAC 1990 (Los Alamos National Laboratory, New Mexico, 1990), p. 232, MO465.

[7] C. Hovater, L. Harwood, and R. Kazimi, The CEBAF RF Separator System (CERN, Geneva, 1996).

[8] B. Xiao, L. Alberty, S. Belomestnykh, I. Ben-Zvi, R. Calaga, C. Cullen, O. Capatina, L. Hammons, Z. Li, C. Marques, J. Skaritka, S. Verdu Andres, and Q. Wu, Design, prototyping, and testing of a compact superconducting double quarter wave crab cavity, Phys. Rev. ST Accel. Beams 18, 041004 (2015).

[9] S. U. De Silva and J. R. Delayen, Cryogenic test of a proofof-principle superconducting rf-dipole deflecting and crabbing cavity, Phys. Rev. ST Accel. Beams 16, 082001 (2013).

[10] S. U. De Silva and J. R. Delayen, Design evolution and properties of superconducting parallel-bar rf-dipole deflecting and crabbing cavities, Phys. Rev. ST Accel. Beams 16, 012004 (2013).

[11] W. K. H. Panofsky and W. A. Wenzel, Some considerations concerning the transverse deflection of charged particles in radio-frequency fields, Rev. Sci. Instrum. 27 (1956), 967.

[12] R. Apsimon and G. Burt, Nucl. Instrum. Methods Phys. Res., Sect. A, doi:10.1016/j.nima.2016.11.030 (2016).

[13] CST Microwave Studio, https://www.cst.com/.

[14] D. R. Brett, R. B. Appleby, R. De Maria, J. B. Garcia, R. T. Garcia, B. Hall, and G. Burt, Accurate crab cavity modeling for the high luminosity Large Hadron Collider, Phys. Rev. ST Accel. Beams 17, 104001 (2014).

[15] J. Barranco Garcia et al., Long term dynamics of the Large Hadron Collider with crab cavity multipoles (to be published).
[16] D. R. Brett, R. B. Appleby, G. Burt, and B. Hall, Particle trajectories in a four rod crab cavity, Nucl. Instrum. Methods Phys. Res., Sect. A 734, 79 (2014).

[17] R. B. Appleby and D. T. Abell, Accurate dynamics in an azimuthally-symmetric accelerating cavity, J. Instrum. 10, P02005 (2015).

[18] P. Baudrenghien, K. Brodzinski, R. Calaga, O. Capatina, E. Jensen, A. Macpherson, E. Montesinos, and V. Parma, Report No. CERN-ACC-NOTE-2013-003, 2013.

[19] I. Maltzer and T. Zitelli, Focus electrode for high power electron guns, US Patent No. 3,281,616 (1966).

[20] R. Calaga, R. De Maria, J. Barranco, M. Giovannozzi, A. Grudiev et al., in Proceedings of the 3rd International Particle Accelerator Conference, New Orleans, LA, 2012 (IEEE, Piscataway, NJ, 2012), p. 1873, TUPPR027.

[21] P. Baudrenghien, LLRF for crab cavities-2012. 2nd Joint HiLumi LHC-LARP Annual meeting, Frascati, Italy (CERN, Geneva, 2012).

[22] W. Davies, The theory of the measurement of magnetic multipole fields with rotating coil magnetometers, Nucl. Instrum. Methods Phys. Res., Sect. A 311, 399 (1992).

[23] L. C. Maier and J. Slater, Field strength measurements in resonant cavities, J. Appl. Phys. 23, 68 (1952).

[24] B. Hall, Ph. D. thesis, Lancaster University, 2014.

[25] P. Baudrenghien et al., Report No. CERN-ACC-NOTE2013-003, 2013.

[26] M. Liu and M. R. Stern, Oscillating Superleak Transducer of Second Sound, Phys. Rev. Lett. 48, 1842 (1982).

[27] Cernox $^{\mathrm{TM}}$ resistance temperature sensors for high energy physics applications, Lake Shore Cryotronics.

[28] A. Macpherson et al., in Proceedings, 17th International Conference on RF Superconductivity (SRF2015): Whistler, Canada, 2015 (TRIUMF, Vancouver, 2015), p. TUPB080.

[29] K. Hernández-Chahín et al., in Proceedings, 17th International Conference on RF Superconductivity (SRF2015): Whistler, Canada, 2015 (TRIUMF, Vancouver, 2015), p. THPB050.

[30] F. Carra et al., in Proceedings, 17th International Conference on RF Superconductivity (SRF2015): Whistler, Canada, 2015 (TRIUMF, Vancouver, 2015), p. FRBA02. 